\title{
Self-Learning Threshold-Based Load Balancing
}

\author{
Diego Goldsztajn, Sem C. Borst \\ Eindhoven University of Technology, d.e.goldsztajn@tue.nl, s.c.borst@tue.nl \\ Johan S.H. van Leeuwaarden \\ Tilburg University, j.s.h.vanleeuwaarden@uvt.nl \\ Debankur Mukherjee \\ Georgia Institute of Technology, debankur.mukherjee@isye.gatech.edu \\ Philip A. Whiting \\ Macquarie University, philip.whiting@mq.edu.au
}

August 19, 2021

\begin{abstract}
We consider a large-scale service system where incoming tasks have to be instantaneously dispatched to one out of many parallel server pools. The user-perceived performance degrades with the number of concurrent tasks and the dispatcher aims at maximizing the overall quality-of-service by balancing the load through a simple threshold policy. We demonstrate that such a policy is optimal on the fluid and diffusion scales, while only involving a small communication overhead, which is crucial for large-scale deployments. In order to set the threshold optimally, it is important, however, to learn the load of the system, which may be unknown. For that purpose, we design a control rule for tuning the threshold in an online manner. We derive conditions which guarantee that this adaptive threshold settles at the optimal value, along with estimates for the time until this happens. In addition, we provide numerical experiments which support the theoretical results and further indicate that our policy copes effectively with time-varying demand patterns.
\end{abstract}

Key words: adaptive load balancing, many-server asymptotics, fluid and diffusion limits. Acknowledgment: the work in this paper is supported by the Netherlands Organisation for Scientific Research (NWO) through Gravitation-grant NETWORKS-024.002.003, by the 
National Science Foundation (NSF) through Grant No. 2113027 and by the Australian

Research Council Discovery Project (DP) through grant DP180103550.

\section{Introduction.}

Consider a service system where incoming tasks have to be immediately routed to one out of many parallel server pools. The service of tasks starts upon arrival and is independent of the number of tasks contending for service at the same server pool. Nevertheless, the portion of shared resources available to individual tasks does depend on the number of contending tasks, and in particular the experienced performance may degrade as the degree of contention rises, creating an incentive to balance the load so as to keep the maximum number of concurrent tasks across server pools as low as possible.

The latter features are characteristic of video streaming applications, such as video conferencing services. In this context, a server pool could correspond to an individual server, instantiated to handle multiple streaming tasks in parallel. The duration of tasks is typically determined by the application and is not significantly affected by the number of instances contending for the finite shared resources of the server (e.g. bandwidth). However, the video and audio quality suffer degradation as these resources get distributed among a growing number of active instances. Effective load balancing policies are thus key to optimizing the overall user experience, but the implementation of these policies must be simple enough as to not introduce significant overheads, particularly in large systems.

As it is standard in the load balancing literature, suppose that the execution times of tasks are exponentially distributed, with unit mean, and that tasks arrive as a Poisson process with aggregate intensity equal to the arrival rate scaled by the number of server pools. The number of tasks in steady state is Poisson distributed with mean equal to this intensity. Thus, a natural and simple load balancing strategy is a threshold policy that assigns incoming tasks to server pools that exhibit a number of tasks smaller than the rounded up arrival rate, if possible. In fact, we prove that a policy of this kind is optimal on the fluid scale: in large-scale systems, the fraction of server pools that feature a number of tasks that exceeds or falls short of the rounded up or down arrival rate vanishes over time; we further show that this policy is still optimal on the more fine-grained diffusion scale and only involves a small communication overhead. To achieve optimality, however, the threshold must be learnt, since it depends on the overall demand for service, which may be unknown or even time-varying. For this purpose, we introduce a control rule for adjusting the threshold in an online fashion, relying solely on the same state information needed to take the dispatching decisions.

Effectively, our adaptive dispatching rule integrates online resource allocation decisions with demand estimation. While these two attributes are evidently intertwined, the online control actions and longer-term estimation rules are typically decoupled and separately 
studied in the literature. The former usually assume perfect knowledge of relevant system parameters, while the latter typically focus on statistical estimation of these parameters. In contrast, our policy smoothly blends these two elements and does not rely on an explicit load estimate, but yields an implicit indication as a by-product.

We analyze this policy theoretically, through fluid and diffusion approximations which are justified by suitable large-scale limit theorems, and also by means of several numerical experiments; our main contributions are listed below in more detail.

- We establish that a threshold policy is optimal on the fluid and the diffusion scales, if the threshold is chosen suitably. Also, we provide a token-based implementation which involves a low communication overhead, of at most two messages per task.

- The optimal threshold depends on the offered load, which tends to be uncertain or even time-varying in practice. We propose a control rule for adjusting the threshold in an online manner to an unknown load, relying solely on the tokens kept at the dispatcher. To analyze this rule we provide a fluid limit for the joint evolution of the system occupancy and the self-learning threshold. To the best of our knowledge, this is the first paper that tackles control parameter adaptations of this kind as part of a fluid-limit analysis.

- We prove that the threshold settles in finite time in a many-server regime, and we provide lower and upper bounds for the equilibrium threshold. These are used to design the control rule to achieve nearly-optimal performance once the threshold has reached an equilibrium. In addition, we derive an upper bound for the limit of the time until the threshold settles as the number of tasks grows large.

- The theoretical results are accompanied by several simulations, which show that the threshold settles in systems with a few hundred servers and only after a short time. Furthermore, even in the presence of highly variable demand patterns, our simulations indicate that the threshold adapts swiftly to variations in the offered load.

Load balancing problems, similar to the one addressed in the present paper, have received a immense attention in the past few decades; [31] provides a recent survey. While traditionally the focus in this literature used to be on performance, more recently the implementation overhead has emerged as an equally important issue. This overhead has two sources: the communication burden of exchanging messages between the dispatcher and the servers, and the cost in large-scale deployments of storing and managing state information at the dispatcher, as considered in [11].

While this paper concerns an "infinite-server" setting, the load balancing literature is predominantly focused on single-server models, where performance is generally measured in terms of queue lengths or delays. In that scenario, the Join the Shortest Queue (JSQ) 
policy minimizes the mean delay for exponentially distributed service times, among all nonanticipating policies; see $[9,34]$. However, a naive implementation of this policy involves an excessive communication burden for large systems. So-called power-of- $d$ strategies assign tasks to the shortest among $d$ randomly sampled queues, which involves substantially less communication overhead and yet provides significant improvements in delay performance over purely random routing, even for $d=2$; see $[21,22,32]$. A further alternative are pullbased policies, which were introduced in $[2,28]$. These policies reduce the communication burden by maintaining state information at the dispatcher. In particular, the Join the Idle Queue (JIQ) policy studied in [19,28] matches the optimality of JSQ in a many-server regime, and involves only one message per task. This is achieved by storing little state information at the dispatcher, in the form of a list of idle queues.

The main differences in the delay performance of the aforementioned policies appear in the heavy-traffic regime where the load approaches one. If we take JSQ as a reference, then JIQ deviates from this benchmark under certain heavy-traffic conditions. This issue was addressed in [37,38], which propose Join Below the Threshold (JBT): a refinement of JIQ for achieving heavy-traffic delay optimality at the expense of increasing the communication overhead only mildly. Despite similarity in name, the problem considered in the latter papers is essentially different from the one addressed in the present paper since achieving delay optimality in a system of parallel single-server queues does not require to maintain a balanced distribution of the load; in fact, the JBT policy does not maintain even queue lengths. The JBT policy was considered for systems of heterogeneous limited processor sharing servers with state-dependent service rates in [15]. Individual limited processor sharing systems with state-dependent service rates were studied in [14], who analyze how to set the multi-programming-limit to minimize the mean response time in a way that is robust with respect to the arrival process; this is a scheduling problem where the way in which the service rate changes with the number of tasks sharing the server is a crucial factor. In the context of purely processor sharing servers with finite buffers, dispatching policies which are insensitive to the job size distribution, as in [5], have received considerable attention. The asymptotic loss probability of an insensitive dispatching policy in a symmetric scenario is studied in [16] and [7] proposes a token-based insensitive load balancing rule for a system with different classes of both jobs and servers, assuming balanced service rates across the server classes.

As mentioned above, the infinite-server setting considered in this paper has received only limited attention in the load balancing literature. While queue lengths and delays are hardly meaningful in this type of scenario, load balancing still plays a crucial role in optimizing different performance measures, and many of the concepts discussed in the single-server context carry over. One relevant performance measure is the loss probability in Erlang-B scenarios. Power-of- $d$ properties for these probabilities have been established in $[17,25,26,30,35]$. Other relevant measures are Schur-concave utility metrics associated 
with quality-of-service as perceived by streaming applications; these metrics are maximized by balancing the load. As in the single-server setting, JSQ is the optimal policy for evenly distributing tasks among server pools, but it involves a significant implementation burden; see $[20,27]$ for proofs of the optimality of JSQ. It was established in [23] that the performance of JSQ can be asymptotically matched by certain power-of- $d$ strategies which reduce the communication overhead significantly, by sampling a suitably chosen number of server pools that depends on the number of tasks and dispatching tasks to the least congested of the sampled server pools.

Just like the strategies studied in [23], the policy considered in this paper aims at optimizing the overall experienced performance and asymptotically matches the optimality of JSQ on the fluid and diffusion scales. Moreover, our policy involves at most two messages per task and requires to store only two tokens per server pool at the dispatcher; from this perspective, our policy is the counterpart of JIQ in the infinite-server setting. Another pull-based strategy in an infinite-server (blocking) scenario was briefly considered in [28]. While this policy minimizes the loss probability, it does not achieve an even distribution of the load and involves storing a larger number of tokens: one for each idle server in the system. From a technical perspective, we use a similar methodology to derive a fluid limit in the case of a static threshold. However, a different proof method is needed when the threshold is adjusted over time.

The infinite-server model considered in this paper represents a scenario with streaming applications that have random but fixed session durations and adaptive resource requirements (e.g. in terms of bandwidth) in the presence of a time-varying number of competing flows. This set-up has been commonly adopted in the literature as a natural modeling paradigm for describing the dynamics and evaluating the performance of streaming sessions on flow-level, see for instance $[3,18]$.

As alluded to above, the most appealing feature of our policy is its capability of adapting the threshold to unknown and time-varying loads. The problem of adaptation to uncertain demand patterns was addressed in the single-server setting in $[12,13,24]$, which remove the classical fixed-capacity assumption of the single-server load balancing literature and assume instead that the number of servers can be adjusted on the fly to match the load. However, in these papers the load balancing policy remains the same at all times since the "right-sizing" mechanism for adjusting the capacity of the system is sufficient to deal with changes in demand. Mechanisms of this kind had already been studied in the singleserver scenario to trade off latency and power consumption in microprocessors; see [33,36]. As in the present paper, all the aforementioned papers attempt to learn the load of the system by observing certain state information; a treatment of the more general problem of estimating parameters of a queueing system by observing its trajectories can be found in [1]. A different option is to forecast demand from historical data, which has been the predominant approach within the call center staffing literature; a recent survey of this 
literature is provided in [8].

Different from any of the above studies, this paper considers a pull-based dispatching policy for optimizing the overall quality-of-service in a system of parallel server pools. This policy is endowed with a self-learning capability which seamlessly adapts to unknown load values in an online manner, and additionally tracks load variations which are prevalent in practice but rarely accounted for in the load balancing literature.

The remainder of the paper is organized as follows. In Section 2 we describe the model and our dispatching policy. In Section 3 we establish that this policy is fluid and diffusion optimal if the threshold is chosen suitably. In Section 4 we analyze a control rule for adjusting the threshold to an unknown load and we explain how to tune this control rule for nearly-optimal performance. Our theoretical results are corroborated by numerical experiments ${ }^{1}$ in Section 5 and conclusions are provided in Section 6. The proofs of some technical results are provided in an online supplement.

\section{Model and Threshold Policy.}

Below we specify a stochastic model for a service system of parallel and identical server pools and we describe a threshold-based load balancing policy for assigning the incoming tasks to the server pools. The model description is carried out in Section 2.1, while the load balancing policy is explained in Section 2.2.

\subsection{Model Description.}

Consider $n$ parallel and identical server pools with infinitely many servers each. Tasks arrive as a Poisson process with rate $n \lambda$, where $\lambda$ is the individual server pool arrival rate, and are immediately routed to one of the server pools, where service starts at once and lasts an exponentially distributed time of unit mean. The execution times are independent of the number of tasks contending for service at the same server pool, but the quality-of-service experienced by tasks degrades as the degree of contention increases. Thus, maintaining an even distribution of the load is key to optimizing the overall quality-of-service.

More specifically, let $X_{i}$ denote the number of concurrent tasks at server pool $i$ and suppose that we resort to a utility metric $u\left(X_{i}\right)=g\left(1 / X_{i}\right)$ as a proxy for measuring the quality-of-service experienced by a task assigned to server pool $i$, as function of its resource share. Provided that $g$ is a concave and increasing function, the overall utility $\sum_{i=1}^{n} X_{i} u\left(X_{i}\right)$ is a Schur-concave function of $X=\left(X_{1}, \ldots, X_{n}\right)$, and is thus maximized by balancing the number of tasks among the various server pools.

The vector-valued process $X$ describing the number of tasks at each of the $n$ server pools

\footnotetext{
${ }^{1}$ All the data depicted in the plots presented in this paper, and the code used to generate this data, are available at https://github.com/diegogolds/self-learning-threshold-policy.
} 


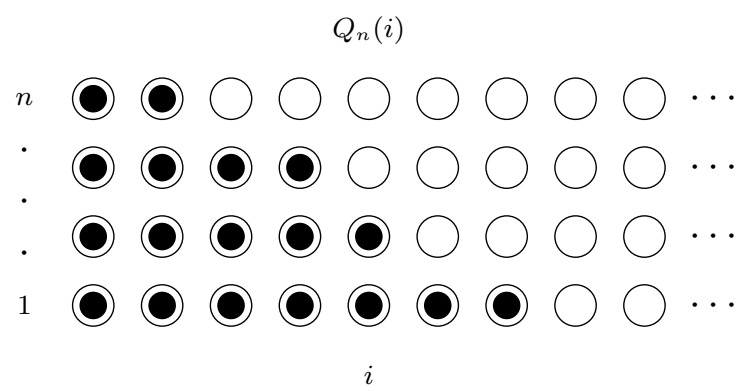

Figure 1: Schematic representation of the system state at a given time. White circles represent servers and black circles represent tasks. Each row corresponds to a server pool, and these are arranged so that the number of tasks increases from top to bottom. The number of tasks in column $i$ equals $Q_{n}(i)$.

constitutes a continuous-time Markov chain when the dispatching decisions are based on the current number of tasks at each server pool. It is, however, more convenient to adopt an aggregate state description, denoting by $Q_{n}(i)$ the number of server pools with at least $i$ tasks, as illustrated in Figure 1. In view of the symmetry of the model, the infinitedimensional process $Q_{n}=\left\{Q_{n}(i): i \geq 0\right\}$ also constitutes a continuous-time Markov chain. We will often consider the normalized processes $q_{n}(i)=Q_{n}(i) / n$ and $q_{n}=\left\{q_{n}(i): i \geq 0\right\}$; the former corresponds to the fraction of server pools with at least $i$ tasks.

\subsection{Threshold-Based Load Balancing Policy.}

All server pools together constitute an infinite-server system, thus the total number of tasks in stationarity is Poisson distributed with mean $n \lambda$. In order to motivate our dispatching rule, let us briefly assume $n \lambda \in \mathbb{N}$. If at a given time the total number of tasks in the system was exactly $n \lambda$, the diagram of Figure 1 would ideally consist of rows with either $\lfloor\lambda\rfloor$ or $\lceil\lambda\rceil$ tasks; where $\lfloor\cdot\rfloor$ and $\lceil\cdot\rceil$ denote the floor and ceiling functions, respectively. This load distribution corresponds to the sequence $q^{*}$ defined as

$$
q^{*}(i)=1 \quad \text { if } \quad i \leq\lfloor\lambda\rfloor, \quad q^{*}(\lfloor\lambda\rfloor+1)=\lambda-\lfloor\lambda\rfloor \quad \text { and } \quad q^{*}(i)=0 \quad \text { if } \quad i>\lfloor\lambda\rfloor+1 .
$$

When the dispatching rule is JSQ, it is shown in [23] that $q_{n}$ has a stationary distribution for each $n$ and that these stationary distributions converge to the Dirac probability measure concentrated at $q^{*}$ as $n \rightarrow \infty$. However, this comes at the expense of a significant communication burden, as observed in the Introduction.

The total number of tasks fluctuates over time, but if tasks are dispatched in a suitable way, it is possible that all server pools have either $\lfloor\lambda\rfloor$ or $\lceil\lambda\rceil$ tasks most of the time, and that only the fraction of server pools with each of these occupancy levels fluctuates. To achieve this we propose a dispatching rule based on a threshold $\ell \in \mathbb{N}$ and the current system occupancy; for brevity we also define $h=\ell+1$. This policy is as follows.

- If $q(\ell)<1$, then some server pool has strictly less than $\ell$ tasks. In this case, new 
tasks are assigned to a server pool with strictly less than $\ell$ tasks, chosen uniformly at random.

- If $q(\ell)=1$ and $q(h)<1$, then all server pools have at least $\ell$ tasks and at least one server pool has exactly $\ell$ tasks. In this case, incoming tasks are sent to a server pool chosen uniformly at random among those with exactly $\ell$ tasks.

- If $q(h)=1$, then all server pools have more than $\ell$ tasks. In this case, new tasks are assigned to a server pool with more than $\ell$ tasks, chosen uniformly at random.

At this point, the main question is if there exists a threshold for which this policy results in an even distribution of the load. Before addressing this question though, we propose a token-based implementation of this policy, which involves a small communication overhead.

In this implementation the dispatcher stores at most two tokens per server pool, labeled 'green' and 'yellow'. A server pool has a green token at the dispatcher if it has strictly less than $\ell$ tasks, and it has a yellow token if it has strictly less than $h$ tasks; note that a server pool with strictly less than $\ell$ tasks will have both a green and a yellow token. When a task arrives to the system, the dispatcher uses these green and yellow tokens as follows.

- In the presence of green tokens, the dispatcher picks one uniformly at random and sends the task to the corresponding server pool; the token is then discarded.

- If the dispatcher only has yellow tokens, one of these tokens is chosen uniformly at random and the task is sent to the corresponding server pool; the token is then discarded.

- In the absence of tokens, the task is sent to a server pool chosen uniformly at random.

In order to maintain accurate state information at the dispatcher, the server pools send messages with updates about their status. A server pool with exactly $h$ tasks which finishes one of its tasks will send a yellow message to the dispatcher, in order to generate a yellow token. Similarly, a server pool with exactly $\ell$ tasks, which finishes one of these tasks, will send a green message to the dispatcher to generate a green token. In addition, green messages are also triggered by arrivals when the number of tasks in the server pool receiving the new task is still strictly less than $\ell$ after the arrival; in this way the green token discarded by the dispatcher is replaced.

With this implementation, a given task may trigger at most two messages: one upon arrival and one after leaving the system; i.e., the communication overhead is at most two messages per task. Also, note that the amount of memory needed at the dispatcher corresponds to $2 n$ tokens. In the next section we will establish that our policy is optimal on the fluid and diffusion scales, for a suitable threshold. This powerful combination of optimality and low communication overhead resembles the properties of JIQ, as considered in the context of single-server queues; see $[19,28]$. 


\section{Optimality Analysis.}

In this section we prove that our policy is optimal on the fluid and diffusion scales, provided that the threshold is chosen suitably. In Section 3.1 we obtain a fluid model of the system, based on a differential equation arising from a fluid limit. This fluid model is used in Section 3.2 to prove that there exist thresholds such that the solutions to the differential equation converge over time to the even distribution of the load $q^{*}$. In particular, we prove that $\ell=\lfloor\lambda\rfloor$ has this property for all $\lambda \geq 0$, and that it is the unique threshold with this property unless $\lambda \in \mathbb{N}$; in the latter special case, $\ell=\lambda-1$ is also fluid-optimal. In Section 3.3 we establish that $\ell=\lfloor\lambda\rfloor$ is optimal on the diffusion scale for all $\lambda \geq 0$.

\subsection{Fluid Limit.}

Next we state a functional strong law of large numbers, also called fluid limit, for the occupancy processes $q_{n}=\left\{q_{n}(i): i \geq 0\right\}$. These processes take values in

$$
Q=\left\{q \in[0,1]^{\mathbb{N}}: q(i+1) \leq q(i) \leq q(0)=1 \text { for all } i \geq 1\right\} \subset \mathbb{R}^{\mathbb{N}}
$$

and their sample paths can be constructed on a common probability space for all $n$, as described in Section A.1 of the online supplement. The space of sample paths over a finite interval of time $[0, T]$ may be endowed with the metric $\rho_{u}^{\infty}$ defined in Section A.2 of the online supplement, which corresponds to uniform convergence in the product topology of $\mathbb{R}^{\mathbb{N}}$. The result stated below holds for any finite time horizon $T$, any threshold $\ell \in \mathbb{N}$ and any random limiting initial condition $q(0)$; a proof is provided in Section A.3 of the online supplement. Informally, this result establishes that the processes $q_{n}$ approach solutions of a certain system of differential equations as $n$ grows large.

Theorem 1. Suppose that $q_{n}(0) \rightarrow q(0)$ in the product topology with probability one, and let $\left.q_{n}\right|_{[0, T]}$ denote the restriction of $q_{n}$ to $[0, T]$. Then $\left\{\left.q_{n}\right|_{[0, T]}: n \geq 1\right\}$ is almost surely relatively compact with respect to the metric $\rho_{u}^{\infty}$. Furthermore, the limit of each convergent subsequence is a function $q:[0, T] \longrightarrow Q$ with Lipschitz coordinates and such that

$$
\dot{q}(i)=\lambda p_{i}(q, \ell)-i[q(i)-q(i+1)] \quad \text { for all } \quad i \geq 1
$$

almost everywhere on $[0, T]$, with respect to the Lebesgue measure. The functions $p_{i}$ in the above differential equations are defined as follows.

(a) If $q(\ell)<1$, then

$$
p_{i}(q, \ell)= \begin{cases}\frac{q(i-1)-q(i)}{1-q(\ell)} & \text { if } \quad 1 \leq i \leq \ell \\ 0 & \text { if } \quad i \geq h\end{cases}
$$


(b) If $q(\ell)=1$ and $q(h)<1$, then

$$
p_{i}(q, \ell)= \begin{cases}\frac{\ell}{\lambda}[1-q(h)] & \text { if } \quad i=\ell \\ 1-\frac{\ell}{\lambda}[1-q(h)] & \text { if } \quad i=h \\ 0 & \text { if } \quad i \neq \ell, h\end{cases}
$$

(c) If $q(h)=1$, then

$$
p_{i}(q, \ell)= \begin{cases}\frac{h}{\lambda}[1-q(h+1)] & \text { if } \quad i=h, \\ {\left[1-\frac{h}{\lambda}(1-q(h+1))\right][q(i-1)-q(i)]} & \text { if } \quad i \geq h+1 \\ 0 & \text { if } \quad 1 \leq i \leq \ell\end{cases}
$$

The fluid dynamics (2) have a simple interpretation: the derivative of $q(i)$ is the rate at which new tasks arrive to server pools with exactly $i-1$ tasks minus the rate at which tasks leave from server pools with precisely $i$ tasks. The term $i[q(i)-q(i+1)]$ corresponds to the cumulative departure rate from server pools with exactly $i$ tasks. Indeed, this quantity equals the total number of tasks in server pools with precisely $i$ tasks and each of these tasks has unit departure rate. The term $p_{i}(q, \ell)$ may be interpreted as the probability that a new task is assigned to a server pool with exactly $i-1$ tasks in fluid state $q$ with threshold $\ell$ in force, and thus $\lambda p_{i}(q, \ell)$ corresponds to the arrival rate of tasks to server pools with $i-1$ tasks. More specifically, the expressions listed in Theorem 1 correspond to the following situations, respectively.

(a) If $q(\ell)<1$, then new tasks are sent to server pools with strictly less than $\ell$ tasks, chosen uniformly at random. So $p_{i}(q, \ell)=0$ if $i \geq h$ and $p_{i}(q, \ell)$ is the fraction of pools with exactly $i-1$ tasks divided by the fraction of pools with at most $\ell-1$ tasks if $1 \leq i \leq \ell$.

(b) If $q(\ell)=1$, then the arrival rate to server pools with precisely $\ell-1$ tasks must equal the departure rate from server pools with exactly $\ell$ tasks, which gives $p_{\ell}(q, \ell)$. If $q(h)<1$ and all server pools have at least $\ell$ tasks, then incoming tasks are sent to server pools with exactly $\ell$ tasks, so $p_{h}(q, \ell)=1-p_{\ell}(q, \ell)$ and $p_{i}(q, \ell)=0$ for all $i \neq \ell, h$.

(c) If $q(h)=1$, then the right-hand side of (2) equals zero for $i=h$, which yields $p_{h}(q, \ell)$. The incoming tasks that are not sent to server pools with exactly $\ell$ tasks are sent to server pools with $h$ or more tasks, and this happens with probability $1-p_{h}(q, \ell)$. Among these server pools, a server pool with exactly $i>h$ tasks is chosen with probability $q(i-1)-q(i)$, which corresponds to uniform random routing. 
The quantity $p_{i}(q, \ell)$ may lie outside $[0,1]$ for some $i, q$ and $\ell$. However, if $q:[0, T] \longrightarrow Q$ is the limit of a convergent subsequence as in Theorem 1 , then $p_{i}(q(t), \ell) \in[0,1]$ for all $i \geq 1$ and all $t \in[0, T]$ outside a set of zero Lebesgue measure.

The proof of Theorem 1 uses a methodology developed in [6] to prove the almost sure relative compactness of sample paths and to establish that the limit of each convergent subsequence has Lipschitz coordinates. These limits are then characterized by a careful analysis in neighborhoods of the points where the derivatives of all coordinates exist, using the stochastic dynamics of the system. The differential equation (2) results from this analysis; details are provided in Section A.3 of the online supplement.

\subsection{Fluid-Optimal Thresholds.}

A threshold $\ell$ is said to be fluid-optimal if all solutions $q:[0, \infty) \longrightarrow Q$ of $(2)$ satisfy

$$
\lim _{t \rightarrow \infty} q(t, i)=q^{*}(i) \quad \text { for all } \quad i \geq 0
$$

recall that $q^{*}$ was defined in (1) and optimizes the overall quality-of-service.

Remark 1. Theorem 1 implies that solutions to (2), defined on $[0, \infty)$, exist for any threshold and initial condition; we do not claim, however, that these solutions are unique.

To identify the fluid-optimal thresholds, we fix $\ell \in \mathbb{N}$ and a solution $q:[0, \infty) \longrightarrow Q$ of (2). We introduce the next functions to analyze how $q$ evolves over time.

Definition 2. Define $u, v_{j}:[0, \infty) \longrightarrow \mathbb{R}$ by $u(t)=\sum_{i=1}^{\infty} q(t, i)$ and $v_{j}(t)=\sum_{i=j}^{\infty} q(t, i)$ for all $j \geq 1$. These are the total mass function and tail mass functions, respectively.

The total mass function $u$ corresponds to the total number of tasks normalized by the number of server pools. The tail mass function $v_{j}$ has a similar interpretation if we visualize tasks as in Figure 1. Namely, it corresponds to the total number of tasks located in column $j$ of the diagram or further to the right, also normalized by the number of server pools.

By taking the sum over $i \geq 1$ on both sides of $(2)$, we obtain $\dot{u}(t)=\lambda-u(t)$ for all $t \geq 0$. This differential equation corresponds to the fluid limit of the total number of tasks in an infinite-server system, which makes obvious sense, reflecting a pre-limit dynamics property. Thus, $u(t)=\lambda+[u(0)-\lambda] \mathrm{e}^{-t}$ for all $t \geq 0$, and in particular $u(t) \rightarrow \lambda$ as $t \rightarrow \infty$. We will use this fact to establish asymptotic upper bounds for $v_{h}(t)$ and $v_{h+1}(t)$ when $h \geq \lambda$.

Proposition 3. Let $x^{+}$denote $\max \{x, 0\}$. If $h>\lambda$, then there exists $t_{0} \geq 0$ such that

$$
\begin{aligned}
& v_{h}(t) \leq(\lambda-\ell)^{+}+\mathrm{e}^{-\left(t-t_{0}\right)}\left[v_{h}\left(t_{0}\right)-(\lambda-\ell)^{+}\right] \\
& v_{h+1}(t) \leq \mathrm{e}^{-\left(t-t_{0}\right)} v_{h+1}\left(t_{0}\right),
\end{aligned}
$$


for all $t \geq t_{0}$. The last inequality also holds if $h \geq \lambda$, with $t_{0}=0$.

Proof. Summing both sides of (2) over $i \geq h+1$, we obtain:

$$
\begin{aligned}
\dot{v}_{h+1}(t) & =[\lambda-h(1-q(t, h+1))] \mathbb{1}_{\{q(t, h)=1\}}-h q(t, h+1)-v_{h+1}(t) \\
& \leq[\lambda-h(1-q(t, h+1))]^{+}-h q(t, h+1)-v_{h+1}(t) \leq(\lambda-h)^{+}-v_{h+1}(t) .
\end{aligned}
$$

If $h \geq \lambda$, then $(\lambda-h)^{+}=0$ and we get (3b) with $t_{0}=0$. If $h>\lambda$, then $q(t, h) \leq u(t) / h$ and $u(t) \rightarrow \lambda$ as $t \rightarrow \infty$ imply that there exists $t_{0} \geq 0$ such that $q(t, h)<1$ for all $t \geq t_{0}$. Similarly to (4), we conclude that

$$
\dot{v}_{h}(t) \leq[\lambda-\ell(1-q(t, h))]^{+}-\ell q(t, h)-v_{h}(t) \leq(\lambda-\ell)^{+}-v_{h}(t) \quad \text { for all } \quad t \geq t_{0} .
$$

This implies (3a), and clearly (3b) also holds.

A consequence of this proposition is that the fraction of server pools with more than $h$ tasks vanishes as $t \rightarrow \infty$ when $h \geq \lambda$. We will use Proposition 3 to prove that, for some suitable values of the threshold, $q(t, i) \rightarrow q^{*}(i)$ as $t \rightarrow \infty$ for all $i$.

Theorem 4. $\ell=\lfloor\lambda\rfloor$ is fluid-optimal for all $\lambda$, and $\ell=\lambda-1$ is fluid-optimal if $\lambda \in \mathbb{N}$.

Proof. Suppose $\ell=\lfloor\lambda\rfloor$. Proposition 3 implies that $v_{h+1}(t) \rightarrow 0$ as $t \rightarrow \infty$, hence

$$
\liminf _{t \rightarrow \infty}\left[q(t, h)+\sum_{i=1}^{\ell} q(t, i)\right]=\lim _{t \rightarrow \infty}\left[u(t)-v_{h+1}(t)\right]=\lim _{t \rightarrow \infty} u(t)=\lambda .
$$

It follows from Proposition 3 that the limit superior of $q(t, h)$ is upper bounded by $\lambda-\lfloor\lambda\rfloor$, and evidently $\sum_{i=1}^{\ell} q(t, i) \leq \ell=\lfloor\lambda\rfloor$. Thus, $q(t, h) \rightarrow \lambda-\lfloor\lambda\rfloor$ and $\sum_{i=1}^{\ell} q(t, i) \rightarrow\lfloor\lambda\rfloor$ as $t \rightarrow \infty$. This implies that $q(t, i) \rightarrow q^{*}(i)$ as $t \rightarrow \infty$ for all $i$. The proof is similar when $\lambda \in \mathbb{N}$ and the threshold is $\ell=\lambda-1$.

The following two examples show that the thresholds mentioned in Theorem 4 are the only thresholds for which all solutions of (2) converge to $q^{*}$. In both cases we exhibit equilibrium solutions of (2) which are different from $q^{*}$.

Example 1 (Large threshold). Suppose $\ell>\lfloor\lambda\rfloor$ and let $\theta \in(0,1)$ be a solution of

$$
\frac{\lambda}{x}=\sum_{i=1}^{\ell} \frac{\ell !}{(\ell-i) !}\left(\frac{1-x}{\lambda}\right)^{i-1} .
$$

Such a solution always exists because the right-hand side has a finite limit as $x \rightarrow 0^{+}$and it converges to $\ell>\lambda$ as $x \rightarrow 1^{-}$. Let us define $q(0) \in Q$ such that $q(0, i)=0$ for all $i>\ell$ and

$$
q(0, \ell-i)-q(0, \ell-(i-1))=\frac{\ell !}{(\ell-i) !}\left(\frac{1-\theta}{\lambda}\right)^{i} \theta \quad \text { for all } \quad 0 \leq i \leq \ell
$$


In order to see that $q(0)$ indeed lies in $Q$, observe that $q(0, \ell)=\theta$ and that

$$
1=\theta+\sum_{i=1}^{\ell} \frac{\ell !}{(\ell-i) !}\left(\frac{1-\theta}{\lambda}\right)^{i} \theta=\sum_{i=0}^{\infty}[q(0, i)-q(0, i+1)]=q(0,0)
$$

where the first equality follows from (5). Also, if we let $\pi(j)=q(0, j)-q(0, j+1)$ for all $0 \leq j \leq \ell$, then $\pi$ is the stationary distribution of an Erlang-B system with $\ell$ servers and offered traffic $\lambda /(1-\theta)$. Moreover, $\theta=q(0, \ell)$ is the blocking probability of this system. Loosely speaking, each server pool behaves as a blocking system with $\ell$ servers and an offered traffic which is larger than $\lambda$, because tasks that find a full server pool are not discarded but sent to a server pool with idle servers.

It is possible to verify that the initial condition $q(0)$ constructed above is a fixed point of $(2)$, so the constant function $q:[0, \infty) \longrightarrow Q$ such that $q(t)=q(0)$ for all $t \geq 0$ solves $(2)$. In addition, note that $q(0)$ corresponds to a suboptimal distribution of the load where the fraction of server pools with exactly $i$ tasks is positive all $0 \leq i \leq \ell$; see Figure 2 .

In the previous example, the maximum number of tasks across server pools is $\ell>\lfloor\lambda\rfloor$. If the goal is to avoid concentrations of tasks, then this situation is nearly-optimal when $\ell$ is close to $\lfloor\lambda\rfloor$; note that the even distribution of the load $q^{*}$ achieved when the threshold $\ell=\lfloor\lambda\rfloor$ is fluid-optimal involves server pools with $\lfloor\lambda\rfloor+1$ tasks. The most problematic situations arise, instead, when the threshold is lower than the optimal value. Intuitively, in these cases all server pools have more than $\ell$ tasks most of the time, because the threshold is smaller than the load. As a result, the system has to resort to random routing very often, which is known to be highly inefficient. In the large-scale limit this translates into the number of tasks across server pools being unbounded.

Example 2 (Small threshold). Suppose that $\ell<\lfloor\lambda\rfloor$ and $\lambda \notin \mathbb{N}$ or, alternatively, that $\ell<\lambda-1$ and $\lambda \in \mathbb{N}$. In addition, let $\theta \in(0,1)$ solve

$$
\sum_{i=h}^{\infty} \frac{h !}{i !}[\lambda-h(1-x)]^{i-h}(1-x)=1
$$

A solution always exists because the left-hand side is strictly larger than one for $x=0$ and equal to zero for $x=1$. We define $q(0) \in Q$ such that $q(0, i)=1$ for all $1 \leq i \leq h$ and

$$
q(0, i)-q(0, i+1)=\frac{h !}{i !}[\lambda-h(1-\theta)]^{i-h}(1-\theta) \quad \text { for all } \quad i \geq h .
$$

By setting $i=h$ in the last equation, we observe that $1-\theta$ is the fraction of server pools with at most $h$ tasks. Furthermore, using (6) we conclude that $\pi(j)=q(0, j)-q(0, j+1)$ for all $j \geq h$ is the stationary distribution of the birth-death process with death rate $j$ at state $j$ and birth rate $\lambda-h(1-\theta)$ at each state. Loosely speaking, tasks are sent to a server pool chosen uniformly at random when all server pools have at least $h$ tasks. The 

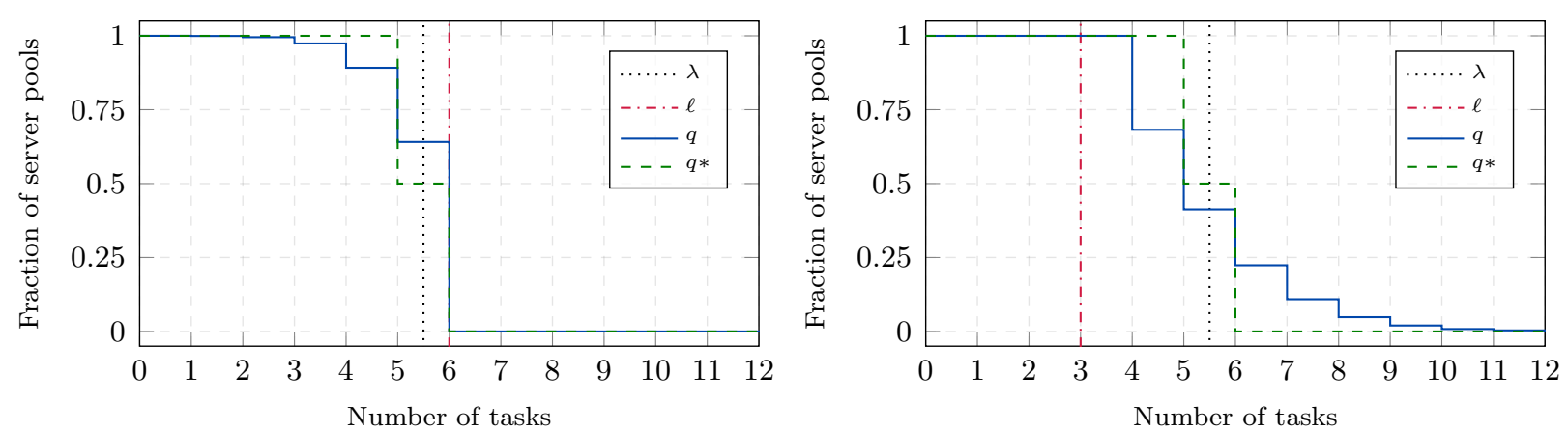

Figure 2: Illustrations of the equilibrium solutions $q$ computed in Examples 1 and 2 for $\lambda=5.5$ and the thresholds indicated in the plots. The left plot corresponds to the case of a large threshold, whereas the right plot corresponds to the case of a small threshold.

fraction of server pools with at least $h$ tasks operates at one, and server pools with exactly $\ell$ tasks receive new tasks at rate $\lambda p_{h}(q(0), \ell)=h(1-\theta)<\lambda$. The remaining tasks are sent to a server pool chosen uniformly at random, so server pools with $h$ or more tasks receive new tasks at rate $\lambda-h(1-\theta)$.

As in Example 1, the initial condition $q(0)$ is a fixed point of (2), so the constant function $q:[0, \infty) \longrightarrow Q$ such that $q(t)=q(0)$ for all $t \geq 0$ solves (2). Also, $q(0)$ is an occupancy state for which the fraction of server pools with exactly $i$ tasks is positive for all $i \geq h$; an occupancy state of this kind is depicted in Figure 2.

The next corollary is a consequence of Theorem 4 and the two examples.

Corollary 5. If $\lambda \notin \mathbb{N}$, then $\lfloor\lambda\rfloor$ is the unique fluid-optimal threshold. In the special case where $\lambda \in \mathbb{N}$ there exist exactly two fluid-optimal thresholds: $\lambda-1$ and $\lambda$.

\subsection{Diffusion-Scale Optimality.}

We have already proved that our policy is fluid-optimal when $\ell=\lfloor\lambda\rfloor$, and we establish here that our policy is optimal on the diffusion scale as well for this value of the threshold. This is done by proving that our policy has the same behavior as JSQ on the diffusion scale; indeed, the optimality properties of JSQ established in [20,27] are stronger than diffusion-scale optimality, and in particular imply that JSQ is optimal on the diffusion scale. Specifically, suppose that $\lambda \notin \mathbb{N}$ and let

$$
\bar{Y}_{n}=\sum_{i=1}^{\lfloor\lambda\rfloor} \frac{n-Q_{n}(i)}{\log n}, \quad \bar{Z}_{n}=\frac{Q_{n}(\lceil\lambda\rceil)-(\lambda-\lfloor\lambda\rfloor) n}{\sqrt{n}}, \quad \bar{Q}_{n}(i)=\frac{Q_{n}(i)}{\sqrt{n}} \quad \text { for all } \quad i>\lceil\lambda\rceil .
$$

These random variables have the same asymptotic behavior both for JSQ and our thresholdbased policy, as stated in the following theorem.

Theorem 6. Suppose $\lambda \notin \mathbb{N}, \ell=\lfloor\lambda\rfloor$ and $\bar{Y}_{n}(0), \bar{Z}_{n}(0)$ and $\bar{Q}_{n}(0, i)$, for all $i>\lceil\lambda\rceil$, have a limit in distribution as $n \rightarrow \infty$; denote these limits by $\bar{Y}(0), \bar{Z}(0)$ and $\bar{Q}(0, i)$, respectively. 
(a) The sequence $\left\{\bar{Y}_{n}: n \geq 1\right\}$ is stochastically bounded in the Skorokhod space $D_{\mathbb{R}}[0, \infty)$.

(b) $\bar{Z}_{n}$ converges weakly in $D_{\mathbb{R}}[0, \infty)$ as $n \rightarrow \infty$ to an Ornstein-Uhlenbeck process $Z$, which satisfies the stochastic differential equation $d Z=-Z d t+\sqrt{2 \lambda} d W$; here $W$ denotes a standard Wiener process.

(c) Provided that $\bar{Q}_{n}(0, i)$ converges in probability to zero, $\bar{Q}_{n}(i)$ converges weakly in $D_{\mathbb{R}}[0, \infty)$ to the identically zero process; this holds for all $i>\lceil\lambda\rceil$.

The proof of Theorem 6 is carried out in Section B.1 of the online supplement through a stochastic coupling between a system which uses our policy and a system which uses the JSQ policy; the diffusion limit of JSQ, which coincides with Theorem 6, was derived in [23]. Loosely speaking, Theorem 6 implies that, for large enough $n$, and after sufficient time, the number of server pools with $\lceil\lambda\rceil$ tasks is $(\lambda-\lfloor\lambda\rfloor) n+O(\sqrt{n})$ and the number of server pools with fewer than $\lfloor\lambda\rfloor$ tasks is $O(\log n)$. Also, if the system starts with no server pools with more than $\lceil\lambda\rceil$ tasks, then it will remain so.

The diffusion scale optimality of the threshold $\ell=\lfloor\lambda\rfloor$ can also be established in the special case $\lambda \in \mathbb{N}$, and this is done in Appendix B of the online supplement.

\section{Learning the Optimal Threshold.}

In this section we propose a control rule for adjusting a dynamic threshold over time, to learn the optimal threshold value when $\lambda$ is unknown. This rule is explained in Section 4.1 and then analyzed through a fluid-limit approach in the following sections. Specifically, a fluid model for systems with dynamic thresholds is introduced in Section 4.2 and is then justified in Section 4.3 through a fluid limit. The evolution of the dynamic threshold over time is analyzed through the fluid model in Section 4.4, where we establish that the threshold always reaches an equilibrium. In Section 4.5 we explain how to tune our learning rule so that the equilibrium threshold is always nearly-optimal and we prove that this tuning yields in fact an optimal equilibrium threshold in most situations. In addition, the time required for the threshold to settle is analyzed in Section 4.6.

\subsection{Learning rule}

In Section 3 we showed that our threshold policy is fluid and diffusion optimal provided that $\ell=\lfloor\lambda\rfloor$. However, these optimality properties critically rely on the threshold being strictly equal to $\lfloor\lambda\rfloor$, as was shown by Examples 1 and 2. Furthermore, in actual system deployments discrepancies between an a priori chosen threshold and the optimal value $\lfloor\lambda\rfloor$ may occur due to the following two reasons. 
1. In general it is difficult to estimate $\lambda$ in advance, and a slightly inaccurate estimate may result in a wrong choice of the threshold. The worst performance repercussions occur if $\lambda$ is underestimated and a low threshold is chosen, as explained before Example 2.

2. Even if the threshold equals $\lfloor\lambda\rfloor$ initially, $\lambda$ could change due to fluctuations in the overall demand for service. These fluctuations could result in a mismatch between $\ell$ and $\lfloor\lambda\rfloor$, with the corresponding adverse consequences in terms of performance.

Remark 2. We have adopted the common assumption of unit-mean service times, which amounts to a convenient choice of time unit. In view of this, it is worth observing that the optimal threshold is determined by the offered load, rather than the arrival rate of tasks. Specifically, if service times had mean $1 / \mu$, then the optimal threshold would be $\ell=\lfloor\rho\rfloor$, with $\rho=\lambda / \mu$ the offered load. In particular, it is the offered load that has to be estimated rather than the arrival rate of tasks, which exacerbates the issues mentioned above. Although we assume unit-mean service times, our results easily generalize to a generic service rate $\mu$ without changing the control rule to be described below, which is designed to track the offered load rather than the arrival rate of tasks.

To achieve optimality it is necessary to actively learn the optimal threshold. To this purpose we propose a control rule for adjusting the threshold in an online fashion. To explain this rule, let us denote the threshold in a system with $n$ server pools by $\ell_{n}(t)$, which is now time-dependent; as before, we also introduce the notation $h_{n}(t)=\ell_{n}(t)+1$ for convenience. Our control rule depends on a parameter $\alpha \in(0,1)$ and it adjusts the threshold only at arrival epochs, right after dispatching the new task. If an arrival occurs at time $\tau$, then the threshold is adjusted as follows.

- The threshold is increased by one if the number of server pools with at least $h_{n}$ tasks, measured right before time $\tau$, is greater than or equal to $n-1$.

- The threshold is decreased by one if the fraction of server pools with at least $\ell_{n}$ tasks, measured right before time $\tau$, is smaller than or equal to $\alpha$.

- Otherwise, the threshold remains unchanged.

This control rule only relies on knowledge of the tokens which are maintained by the dispatcher when the implementation of Section 2.2 is adopted. Specifically, the threshold is increased if and only if the number of yellow tokens was smaller than or equal to one prior to the arrival, and the threshold is decreased if and only if the number of green tokens was larger than or equal to $(1-\alpha) n$ right before the arrival occurred.

Suppose $\lambda$ is unknown, either because it was not possible to estimate the offered load in advance or because it recently changed. As tasks arrive to the system, the control rule 
that we have just described adjusts the threshold in steps of one unit. At this point the most relevant question is whether it is possible to choose $\alpha$ so that these updates eventually cease, with the threshold settling at the optimal value. Another important question is how long it takes for the threshold to settle. Indeed, after each update the new threshold must be communicated to all server pools and the information stored at the dispatcher must be updated. Fast convergence of the threshold is thus desired.

\subsection{Fluid Systems.}

Below we introduce the notion of a fluid system, which will help us model the behavior of large-scale systems that use our adaptive threshold policy through differential equations.

Definition 7. Consider sequences $\left\{\tau_{j}: 0 \leq j<\eta\right\}$ and $\left\{\ell_{j} \in \mathbb{N}: 0 \leq j<\eta\right\}$ of increasing times and thresholds, respectively. Suppose $\tau_{0}=0$, let $\tau_{\eta}=\infty$ if $\eta<\infty$ and $\tau_{\eta}=\lim _{j \rightarrow \infty} \tau_{j}$ otherwise. We define $\ell:\left[0, \tau_{\eta}\right) \longrightarrow \mathbb{N}$ such that $\ell(t)=\ell_{j}$ for all $t \in\left[\tau_{j}, \tau_{j+1}\right)$ and all $0 \leq j<\eta$, and given $q:\left[0, \tau_{\eta}\right) \longrightarrow Q$, we say that $s=(q, \ell)$ is a fluid system if the following hold.

(a) $q(t, \ell(t)) \geq \alpha$ for all $t \in\left[0, \tau_{\eta}\right)$ and $q(h)<1$ almost everywhere on $\left[0, \tau_{\eta}\right)$ with respect to the Lebesgue measure; as usual we define $h_{j}=\ell_{j}+1$ and $h(t)=\ell(t)+1$.

(b) Either $q\left(\tau_{j+1}, \ell_{j}\right)=\alpha$, or alternatively, $q\left(\tau_{j+1}, h_{j}\right)=1$ for all $0 \leq j<\eta-1$. Moreover,

$$
\ell_{j+1}=\ell_{j}-1 \quad \text { if } \quad q\left(\tau_{j+1}, \ell_{j}\right)=\alpha \quad \text { and } \quad \ell_{j+1}=\ell_{j}+1 \quad \text { if } \quad q\left(\tau_{j+1}, h_{j}\right)=1
$$

(c) The coordinate functions $q(i)$ are absolutely continuous and

$$
\dot{q}(i)=\lambda p_{i}\left(q, \ell_{j}\right)-i[q(i)-q(i+1)] \quad \text { for all } \quad i \geq 1
$$

almost everywhere on $\left[\tau_{j}, \tau_{j+1}\right)$ for all $0 \leq j<\eta$.

A fluid system consists of a function $q$, which represents the system occupancy, and a piecewise constant function $\ell$, which represents the time-dependent threshold; the times $\tau_{j}$ correspond to threshold updates. Between $\tau_{j}$ and $\tau_{j+1}$ the threshold is constant, equal to $\ell_{j}$, and the system behaves according to the differential equation of Theorem 1 . In addition, the threshold is updated according to the control rule explained above: informally, the threshold increases when $q(h)$ reaches one and it decreases when $q(\ell)$ drops below $\alpha$.

In Definition 7 , the possibly finite $\tau_{\eta}$ accounts for the possibility of infinitely many updates in finite time; we will prove, however, that this in fact cannot happen. To this end we resort to the total mass function $u:\left[0, \tau_{\eta}\right) \longrightarrow \mathbb{R}$ of Definition 2. As in Section 3.2,

$$
u(t)=\lambda+[u(0)-\lambda] \mathrm{e}^{-t} \text { for all } t \in\left[0, \tau_{\eta}\right)
$$


Indeed, for each $0 \leq j<\eta$ we see that $\dot{u}(t)=\lambda-u(t)$ for all $t \in\left[\tau_{j}, \tau_{j+1}\right)$ by taking the sum over $i \geq 1$ on both sides of (8). The following proposition establishes that the threshold of a fluid system cannot change infinitely many times in finite time.

Proposition 8. All fluid systems have $\tau_{\eta}=\infty$.

Proof. Consider a fluid system with $\eta=\infty$, otherwise the claim holds by Definition 7. It follows from (9) that $u(t)$ is upper bounded by some constant $M \geq 0$. Since the sequence $q(t) \in Q$ is non-increasing for each time $t$, we have $\alpha \leq q\left(t, \ell_{j}\right) \leq u(t) / \ell_{j} \leq M / \ell_{j}$ for all $t \in\left[\tau_{j}, \tau_{j+1}\right)$ and all $j \geq 0$. Therefore, $\left\{\ell_{j}: j \geq 0\right\}$ is a bounded set and, as a result, the set $\mathcal{L}=\left\{\ell \in \mathbb{N}: \ell_{j}=\ell\right.$ for infinitely many $\left.j\right\}$ is non-empty and bounded. We define $m=\max \mathcal{L}$ and we observe that there exists $j_{0} \geq 0$ such that $\ell_{j} \leq m$ for all $j \geq j_{0}$.

Fix any index $j>j_{0}$ such that $\ell_{j}=m$. By (8) we know that

$$
\dot{q}(m)=\lambda p_{m}\left(q, \ell_{j}\right)-m[q(m)-q(m+1)] \geq-m
$$

almost everywhere on $\left[\tau_{j}, \tau_{j+1}\right)$. The thresholds $\ell_{j-1}$ and $\ell_{j+1}$ are equal to $m-1$ by definition of $j_{0}$. In particular, $q\left(\tau_{j}, m\right)=1$ and $q\left(\tau_{j+1}, m\right)=\alpha$, which implies that

$$
\alpha=q\left(\tau_{j+1}, m\right) \geq q\left(\tau_{j}, m\right)-m\left(\tau_{j+1}-\tau_{j}\right)=1-m\left(\tau_{j+1}-\tau_{j}\right) .
$$

By definition of $m$ there exist infinitely many indexes $j>j_{0}$ such that $\ell_{j}=m$. For these indexes we have proved that $\tau_{j+1}-\tau_{j} \geq(1-\alpha) / m$, and therefore $\tau_{j} \rightarrow \infty$ as $j \rightarrow \infty$.

\subsection{Fluid Limit.}

Next we provide a fluid limit which justifies the use of fluid systems as an asymptotic approximation to the behavior of the discrete system $\left(q_{n}, \ell_{n}\right)$ as $n$ grows large. Before stating this fluid limit we must introduce a few technical definitions. Namely, for each finite time horizon $T$ consider the space $D_{\mathbb{R}}[0, T]$ of all real càdlàg functions on $[0, T]$, and endow it with the metric $\rho_{s}$ defined in [4, Section 12], which is complete and corresponds to the Skorokhod $J_{1}$-topology. In addition, recall the metric $\rho_{u}^{\infty}$ mentioned in Section 3.1 and defined rigorously in Section A.2 of the online supplement; this metric is defined on the Skorokhod space $D_{\mathbb{R}^{\mathbb{N}}}[0, T]$ of all càdlàg functions with values in $\mathbb{R}^{\mathbb{N}}$.

For each discrete system $s_{n}=\left(q_{n}, \ell_{n}\right)$, the restriction $\left.s_{n}\right|_{[0, T]}$ is a random function lying in the space $S=D_{\mathbb{R}^{\mathbb{N}}}[0, T] \times D_{\mathbb{R}}[0, T]$, which we endow with the following metric:

$$
\varrho((p, x),(q, y))=\max \left\{\rho_{u}^{\infty}(p, q), \rho_{s}(x, y)\right\} .
$$

Observe that a sequence $\left\{\left(p_{n}, x_{n}\right) \in S: n \geq 1\right\}$ converges with respect to $\varrho$ if and only if $p_{n}$ converges uniformly over $[0, T]$, with respect to the product topology of $\mathbb{R}^{\mathbb{N}}$, and $x_{n}$ converges in the Skorokhod $J_{1}$-topology. 
As in Section 3.1, it is possible to construct the sample paths of $s_{n}$ on a common probability space for all $n$. We adopt such a construction to state the next result, which holds for any pair of random elements $q(0)$ and $\ell(0)$ taking values in $Q$ and $\mathbb{N}$, respectively.

Theorem 9. Suppose $q_{n}(0) \rightarrow q(0)$ in the product topology and $\ell_{n}(0) \rightarrow \ell(0)$ almost surely as $n \rightarrow \infty$. Then $\left\{\left.s_{n}\right|_{[0, T]}: n \geq 1\right\}$ is almost surely relatively compact with respect to $\varrho$ and the limit $s \in S$ of each convergent subsequence can be extended to a fluid system.

The almost sure relative compactness of $\left\{\left.q_{n}\right|_{[0, T]}: n \geq 1\right\}$ with respect to $\rho_{u}^{\infty}$ can be obtained using the methodology of [6], as indicated in Section 3.1. Given a sample path such that the latter relative compactness holds, the challenge is to demonstrate that, for each convergent subsequence $\left\{\left.q_{n_{k}}\right|_{[0, T]}: k \geq 1\right\}$, the associated thresholds converge with respect to $\rho_{s}$, and to characterize the limits of $\left.q_{n_{k}}\right|_{[0, T]}$ and $\left.\ell_{n_{k}}\right|_{[0, T]}$ jointly. This is done inductively, by approaching $s_{n}$ by systems where only finitely many threshold updates occur, starting with systems where the threshold is constant over time as in Section 3.1. The proof of this result is provided in Section A.4 of the online supplement.

\subsection{Convergence of the Threshold.}

In the statement of Theorem 9, the limit $s$ of a convergent subsequence is the restriction to the interval $[0, T]$ of a fluid system. Next we will show that the time-dependent threshold of any fluid system eventually settles at an equilibrium value. For this purpose, we fix a fluid system $s=(q, \ell)$ and we consider the associated total mass and tail mass functions, as in Definition 2. The next result provides upper bounds for the tail mass functions.

Proposition 10. Suppose there exist $m \geq 0$ and $0 \leq a<b$ such that $\ell(t) \leq m$ for all $t \in(a, b)$. Then the following inequalities hold for all $t \in[a, b]$.

$$
\begin{aligned}
& v_{m+1}(t) \leq(\lambda-m)^{+}+\mathrm{e}^{-(t-a)}\left[v_{m+1}(a)-(\lambda-m)^{+}\right] \\
& v_{m+2}(t) \leq \mathrm{e}^{-(t-a)} v_{m+2}(a)
\end{aligned}
$$

If in addition $q(t, m)<1$ for all $t \in(a, b)$, then

$$
v_{m+1}(t) \leq \mathrm{e}^{-(t-a)} v_{m+1}(a) \text { for all } t \in[a, b] .
$$

Proof. Recall from Definition 7 that $q(h)<1$ almost everywhere. For each $t \in(a, b)$ we have $h(t) \leq m+1$, so $\lambda p_{m+1}(s) \leq[\lambda-m(1-q(m+1))]^{+}$and $p_{i}(s)=0$ for all $i \geq m+2$ almost everywhere on $(a, b)$. The proof of (10) proceeds as in Proposition 3 and (11) follows similarly, by noting that $q(m)<1$ implies that $p_{i}(s)=0$ for all $i \geq m+1$.

We now prove that the threshold settles; the next result holds for all $\lambda \geq 0$.

Theorem 11. There exist $t_{\mathrm{eq}} \geq 0$ and $\ell_{\mathrm{eq}} \in \mathbb{N}$ such that $\ell(t)=\ell_{\mathrm{eq}}$ for all $t \geq t_{\mathrm{eq}}$. 
Proof. By (9) there exists $t_{0} \geq 0$ such that $u(t)<\lfloor\lambda\rfloor+1$ for all $t \geq t_{0}$. Hence,

$$
\tau_{j} \geq t_{0} \quad \text { and } \quad \ell_{j} \geq\lfloor\lambda\rfloor \quad \text { imply } \quad q\left(t, h_{j}\right) \leq \frac{u(t)}{h_{j}}<1 \quad \text { for all } \quad t \in\left[\tau_{j}, \tau_{j+1}\right) .
$$

Hence, one of the next situations occurs: the next threshold update happens at $\tau_{j+1}<\infty$ and it corresponds to a threshold decrease, or no further updates occur and $\tau_{j+1}=\infty$.

We now consider two alternative scenarios. First, suppose $\ell(t) \geq\lfloor\lambda\rfloor$ for all $t \geq t_{0}$. The previous observation implies $\ell$ is non-increasing, integer-valued and lower bounded along the interval $\left[t_{0}, \infty\right)$. Therefore, $\ell(t)$ settles at some $\ell_{\text {eq }} \geq\lfloor\lambda\rfloor$. Alternatively, assume there exists $t_{1} \geq t_{0}$ such that $\ell\left(t_{1}\right)<\lfloor\lambda\rfloor$. If $\ell$ reaches $\lfloor\lambda\rfloor$ after $t_{1}$, then $\ell$ cannot increase any further by $(12)$, so $\ell(t) \leq\lfloor\lambda\rfloor$ for all $t \geq t_{1}$.

In the latter case, Proposition 10 holds with $m=\lfloor\lambda\rfloor, a=t_{1}$ and $b=\infty$. In particular,

$$
v_{\lfloor\lambda\rfloor+1}(t) \leq \lambda-\lfloor\lambda\rfloor+\left[v_{\lfloor\lambda\rfloor+1}\left(t_{1}\right)-(\lambda-\lfloor\lambda\rfloor)\right] \mathrm{e}^{-\left(t-t_{1}\right)} \quad \text { for all } \quad t \geq t_{1} .
$$

The right-hand side converges to $\lambda-\lfloor\lambda\rfloor$ over time, and $u(t) \rightarrow \lambda$ over time by (9). As a result, there exists $t_{2} \geq t_{1}$ such that $u(t)-v_{\lfloor\lambda\rfloor+1}(t)>\lfloor\lambda\rfloor-(1-\alpha)$ for all $t \geq t_{2}$.

Suppose that $\tau_{j} \geq t_{2}$, then $q\left(t, \ell_{j}\right) \geq u(t)-(\lfloor\lambda\rfloor-1)-v_{\lfloor\lambda\rfloor+1}(t)>\alpha$ for all $t \in\left[\tau_{j}, \tau_{j+1}\right)$, because $\ell_{j} \leq\lfloor\lambda\rfloor$. This implies that one of the following two situations occurs: the next threshold update happens at $\tau_{j+1}<\infty$ and it is a threshold increase, or no further updates occur and $\tau_{j+1}=\infty$.

Concluding, if $\ell\left(t_{1}\right)<\lfloor\lambda\rfloor$ for some $t_{1} \geq t_{0}$, then there exists $t_{2} \geq t_{1}$ such that the threshold is non-decreasing along the interval $\left[t_{2}, \infty\right)$ and is bounded above by $\lfloor\lambda\rfloor$. Thus, $\ell$ settles in this case as well, at some value $\ell_{\mathrm{eq}} \leq\lfloor\lambda\rfloor$.

\subsection{Tuning of the Learning Rule.}

Theorem 11 does not provide any information about $\ell_{\mathrm{eq}}$ or $t_{\mathrm{eq}}$. Particularly, we would like to know how these values depend on $\alpha$, to set this parameter in a suitable manner. To shed some light on this matter, we first investigate the possible values of $\ell_{\text {eq }}$. We provide lower and upper bounds, which are in turn used to obtain a criterion for setting $\alpha$.

Proposition 12. If $\lambda \notin \mathbb{N}$ then $\ell_{\mathrm{eq}} \geq\lfloor\lambda\rfloor$, and if $\lambda \in \mathbb{N}$ then $\ell_{\mathrm{eq}} \geq \lambda-1$.

Proof. Let us define $h_{\mathrm{eq}}=\ell_{\mathrm{eq}}+1$ and suppose $\lambda \notin \mathbb{N}$; the proof is similar if $\lambda \in \mathbb{N}$. Proposition 10 , with $m=\ell_{\mathrm{eq}}, a=t_{\mathrm{eq}}$ and $b=\infty$, implies that $v_{h_{\mathrm{eq}}+1}(t) \leq v_{h_{\mathrm{eq}}+1}\left(t_{\mathrm{eq}}\right) \mathrm{e}^{-\left(t-t_{\mathrm{eq}}\right)}$ for all $t \geq t_{\text {eq }}$. This in turn implies that $u(t)-v_{h_{\text {eq }}+1}(t) \rightarrow \lambda$ over time. As a result, we have

$$
h_{\mathrm{eq}} \geq \limsup _{t \rightarrow \infty} \sum_{i=1}^{h_{\mathrm{eq}}} q(t, i)=\lim _{t \rightarrow \infty}\left[u(t)-v_{h_{\mathrm{eq}}+1}(t)\right]=\lambda \text {. }
$$

Since $\lambda \notin \mathbb{N}$, we conclude that $h_{\mathrm{eq}} \geq\lceil\lambda\rceil$ and thus $\ell_{\mathrm{eq}} \geq\lfloor\lambda\rfloor$. 
Proposition 13. The equilibrium threshold satisfies $\ell_{\mathrm{eq}} \leq \lambda / \alpha$, both for $\lambda \notin \mathbb{N}$ and $\lambda \in \mathbb{N}$.

Proof. Recall from the proof of Proposition 12 that $u(t)-v_{h_{\mathrm{eq}}+1}(t) \rightarrow \lambda$ as $t \rightarrow \infty$. Also, $q(t, i) \geq q\left(t, \ell_{\mathrm{eq}}\right) \geq \alpha$ for all $t \geq t_{\mathrm{eq}}$ and all $i \leq \ell_{\mathrm{eq}}$ by Definition 7 . Consequently,

$$
0 \leq \limsup _{t \rightarrow \infty} q\left(t, h_{\mathrm{eq}}\right)=\limsup _{t \rightarrow \infty}\left[u(t)-v_{h_{\mathrm{eq}}+1}(t)-\sum_{i=1}^{\ell_{\mathrm{eq}}} q(t, i)\right] \leq \lambda-\alpha \ell_{\mathrm{eq}}
$$

and this completes the proof.

Assume an upper bound of $\lambda$ is known, say $\lambda_{\max }$. We propose to set $\alpha$ such that

$$
\alpha>\frac{\lambda_{\max }}{\lambda_{\max }+1}
$$

the assumption that $\lambda \leq \lambda_{\max }$ is not strong in practice, especially since the above criterion can be used even if the upper bound $\lambda_{\max }$ is chosen conservatively.

The right-hand side of (13) is increasing in $\lambda_{\max }$, which implies that $\alpha>\lambda /(\lambda+1)$ for all offered loads $\lambda \leq \lambda_{\text {max }}$. Recall that $\ell_{\mathrm{eq}} \leq \lambda / \alpha<\lambda+1$ by Proposition 13 , thus $\ell_{\mathrm{eq}} \leq\lceil\lambda\rceil$ for all $\lambda$; i.e., the equilibrium threshold is at most the optimal threshold plus one. More specifically, $\lfloor\lambda\rfloor \leq \ell_{\mathrm{eq}} \leq\lceil\lambda\rceil$ if $\lambda \notin \mathbb{N}$ and $\lambda-1 \leq \ell_{\mathrm{eq}} \leq \lambda$ in the special case $\lambda \in \mathbb{N}$.

We claim that this criterion guarantees nearly-optimal behavior on the fluid scale. To see why, observe that $\ell_{\mathrm{eq}} \leq\lceil\lambda\rceil$. Thus, Proposition 10, with $m=\lceil\lambda\rceil, a=t_{\text {eq }}$ and $b=\infty$, implies that $v_{\lfloor\lambda\rfloor+2}(t) \leq e^{-\left(t-t_{\mathrm{eq}}\right)} v_{\lfloor\lambda\rfloor+2}\left(t_{\mathrm{eq}}\right)$ for all $t \geq t_{\text {eq }}$. Hence, after the threshold settles, the fraction of server pools with more than $\lfloor\lambda\rfloor+1$ tasks decays at least exponentially fast to zero. Although the system may not attain the ideal distribution of the load defined in (1), the fraction of server pools with $\lceil\lambda\rceil$ tasks or more vanishes over time.

If (13) holds, then the threshold settles at a nearly-optimal value. However, in many situations the threshold will in fact settle at the optimal value. This is established in the next corollary, which is a straightforward consequence of Propositions 12 and 13.

Corollary 14. Suppose that

$$
\frac{\lambda}{\lfloor\lambda\rfloor+1}<\alpha
$$

This condition implies that $\ell_{\mathrm{eq}}=\lfloor\lambda\rfloor$ if $\lambda \notin \mathbb{N}$ or $\ell_{\mathrm{eq}} \in\{\lambda-1, \lambda\}$ if $\lambda \in \mathbb{N}$.

This corollary tells us that the optimality of the equilibrium threshold may be lost only when $\lambda$ is close enough to an integer from below. For each $\alpha$ it is possible to find values of $\lambda$ which violate (13). However, the set of such $\lambda$ decreases to the empty set as $\alpha \rightarrow 1$. 


\subsection{Convergence Time.}

Assuming that $\lambda \notin \mathbb{N}$ and that the optimality condition (14) holds, we now focus on the asymptotic time $t_{\text {eq }}$ required by our learning rule to reach an equilibrium. In particular, the next proposition provides an upper bound $\bar{t}_{\mathrm{eq}}$ for this time.

Proposition 15. Suppose $\lambda \notin \mathbb{N}$ and (14) holds. Then, for all

$$
t \geq \bar{t}_{\mathrm{eq}}=\left\{\begin{array}{lll}
\log \left(\frac{\lambda}{\lambda-\lfloor\lambda\rfloor}\right) & \text { if } & u(0) \leq \lambda \\
{\left[\log \left(\frac{u(0)-\lambda}{\alpha[\lambda]-\lambda}\right)\right]^{+}+\log \left(\frac{\lambda}{\lambda-\lfloor\lambda\rfloor}\right)} & \text { if } & u(0)>\lambda
\end{array}\right.
$$

we have $\ell(t)=\lfloor\lambda\rfloor$ and $q(t,\lfloor\lambda\rfloor)=1$. In particular, $\ell$ settles at $t_{e q} \leq \bar{t}_{\text {eq }}$.

Proof. Similarly to (12), we may write

$$
q\left(t, \ell_{j}\right) \leq \frac{u(t)}{\ell_{j}}=\frac{\lambda+[u(0)-\lambda] \mathrm{e}^{-t}}{\ell_{j}} \quad \text { for all } t \in\left[\tau_{j}, \tau_{j+1}\right) \quad \text { and } \quad 0 \leq j<\eta
$$

where we used (9) for the last step. We now choose $s_{0}$ so that the right-hand side is strictly less than $\alpha$ if $\ell_{j} \geq\lceil\lambda\rceil$ and $t>s_{0}$. Specifically, using (14) we set

$$
s_{0}=0 \quad \text { if } \quad u(0) \leq \lambda \quad \text { and } \quad s_{0}=\left[\log \left(\frac{u(0)-\lambda}{\alpha\lceil\lambda\rceil-\lambda}\right)\right]^{+} \quad \text { if } \quad u(0)>\lambda .
$$

Note that $\ell(t) \leq\lfloor\lambda\rfloor$ for all $t>s_{0}$ necessarily. To see why, recall that Definition 7 imposes $q(\ell) \geq \alpha$ at all times, and then note that $\ell(t) \geq\lceil\lambda\rceil$ and $t>s_{0}$ would imply $q(t, \ell(t))<\alpha$. In the special case $\lfloor\lambda\rfloor=0$, this observation implies that $\ell(t)=\lfloor\lambda\rfloor$ for all $t>s_{0}$. In particular, $\ell(t)=\lfloor\lambda\rfloor$ and $q(t,\lfloor\lambda\rfloor)=1$ (trivially) for all $t \geq \bar{t}_{\text {eq. }}$. Thus, we assume below that $\lfloor\lambda\rfloor>0$.

Let $w=u-v_{\lceil\lambda\rceil}$. Summing over $1 \leq i \leq\lfloor\lambda\rfloor$ on both sides of (8), we obtain

$$
\dot{w}(t)=\sum_{i=1}^{\lfloor\lambda\rfloor} \dot{q}(t, i) \geq \lambda-\lfloor\lambda\rfloor>0 \quad \text { whenever } \quad \ell(t) \leq\lfloor\lambda\rfloor \quad \text { and } \quad q(t,\lfloor\lambda\rfloor)<1,
$$

which implies that $w$ is non-decreasing after $s_{0}$. Indeed, $\ell(t) \leq\lfloor\lambda\rfloor$ after $s_{0}$, so $\dot{w}$ is positive unless $q(\lfloor\lambda\rfloor)=1$, which is equivalent to $w$ attaining its maximum value $\lfloor\lambda\rfloor$.

Suppose $q(t,\lfloor\lambda\rfloor)<1$ for all $t \in\left[s_{0}, \bar{t}_{\mathrm{eq}}\right]$. This implies that (11) holds with $m=\lfloor\lambda\rfloor$, $a=s_{0}$ and $b=\bar{t}_{\text {eq }}$. Using (9) as well, we arrive at the following contradiction:

$$
w\left(\bar{t}_{\mathrm{eq}}\right)=u\left(\bar{t}_{\mathrm{eq}}\right)-v_{\lceil\lambda\rceil}\left(\bar{t}_{\mathrm{eq}}\right) \geq \lambda+\left[u\left(s_{0}\right)-\lambda\right] \mathrm{e}^{-\left(\bar{t}_{\mathrm{eq}}-s_{0}\right)}-v_{\lceil\lambda\rceil}\left(s_{0}\right) \mathrm{e}^{-\left(\bar{t}_{\mathrm{eq}}-s_{0}\right)} \geq\lfloor\lambda\rfloor .
$$

Thus, there exists $s_{1} \in\left[s_{0}, \bar{t}_{\text {eq }}\right]$ such that $q\left(s_{1},\lfloor\lambda\rfloor\right)=1$. Since $w\left(s_{1}\right)=\lfloor\lambda\rfloor$ is at its maximum, $q(\lfloor\lambda\rfloor)$ cannot decrease. This implies that $\ell(t)=\lfloor\lambda\rfloor$ and $q(t,\lfloor\lambda\rfloor)=1$ for all $t \geq \bar{t}_{\mathrm{eq}}$ 
The expressions for $\bar{t}_{\text {eq }}$ provided in (15) consist of two terms: the first one upper bounds the time until the threshold falls and remains below $\lfloor\lambda\rfloor$, and the second one accounts for the additional amount of time until the threshold reaches $\lfloor\lambda\rfloor$ and settles. The first term is zero when $u(0) \leq \lambda$, in this case $\ell$ can never exceed $\lfloor\lambda\rfloor$ since $u$ is upper bounded by $\lambda$; here the expression in (15) corresponds to the time needed by $u$ to reach $\lfloor\lambda\rfloor$ when $u(0)=0$. When $u(0)>\lambda$ the two terms are positive. The first one increases with the initial total mass, as one would expect, and, more interestingly, also depends on $\alpha\lceil\lambda\rceil-\lambda$. Loosely speaking, if the fractional part of $\lambda$ is large then it might take longer for $q(\lceil\lambda\rceil)$ to drop below $\alpha$, to trigger a threshold update from $\lceil\lambda\rceil$ to $\lfloor\lambda\rfloor$. The second term is the same as in the case $u(0) \leq \lambda$ and could possibly be reduced.

The next corollary uses the upper bound $\bar{t}_{\text {eq }}$ to summarize the asymptotic optimality properties of our policy when $\lambda \notin \mathbb{N}$ and (14) holds. Informally, this corollary states that the threshold settles at the optimal value $\lfloor\lambda\rfloor$ before $\bar{t}_{\text {eq }}$ in all sufficiently large systems and that the occupancy approaches $q^{*}$ over time at least exponentially fast. The proof is a straightforward consequence of Theorem 9 and Propositions 10 and 15.

Corollary 16. Suppose $\lambda \notin \mathbb{N}$ and (14) holds. There exists a constant $c>0$ such that

$$
\begin{aligned}
& \lim _{n \rightarrow \infty} \sup _{t \in\left[\bar{t}_{\mathrm{eq}}, T\right]}\left|\ell_{n}(t)-\lfloor\lambda\rfloor\right|=0, \\
& \lim _{n \rightarrow \infty} \sup _{t \in\left[\bar{t}_{\mathrm{eq}}, T\right]}\left|q_{n}(t, i)-q^{*}(i)\right|=0 \quad \text { for all } i \leq\lfloor\lambda\rfloor, \\
& \limsup _{n \rightarrow \infty} \sup _{t \in\left[\bar{t}_{\mathrm{eq}}, T\right]}\left|q_{n}(t, i)-q^{*}(i)\right| e^{t-\bar{t}_{\mathrm{eq}}} \leq c \text { for all } i \geq\lceil\lambda\rceil,
\end{aligned}
$$

almost surely for all $T \geq \bar{t}_{\text {eq }}$; and $c$ may be expressed in terms of $\alpha, \lambda$ and $u(0)$ alone.

\section{Simulations.}

In this section we investigate the threshold policy through several numerical experiments $^{2}$. The first set of experiments evaluates the fluid and diffusion approximations suggested by our theoretical results. Next, we examine the user-perceived performance of several dispatching policies, including the threshold policy; we present a histogram of the empirical distribution of the fraction of resources received by a user in stationarity. Finally, we show that the threshold policy copes effectively with highly variable demand patterns.

First, we investigate how large $n$ must be for $\ell_{n}$ to settle, as stated in Theorem 11 for the fluid limit. To this end we consider systems with $\lambda=2.9$ and different values of $n$; in Figure 3 we plot trajectories of two of these systems. In the system with $n=100$ the threshold hovers around the optimal value, oscillating between $\lfloor\lambda\rfloor-1=1$ and $\lceil\lambda\rceil=3$.

\footnotetext{
${ }^{2}$ All the data depicted in the plots presented in this section, and the code used to generate this data, are available at https://github.com/diegogolds/self-learning-threshold-policy.
} 

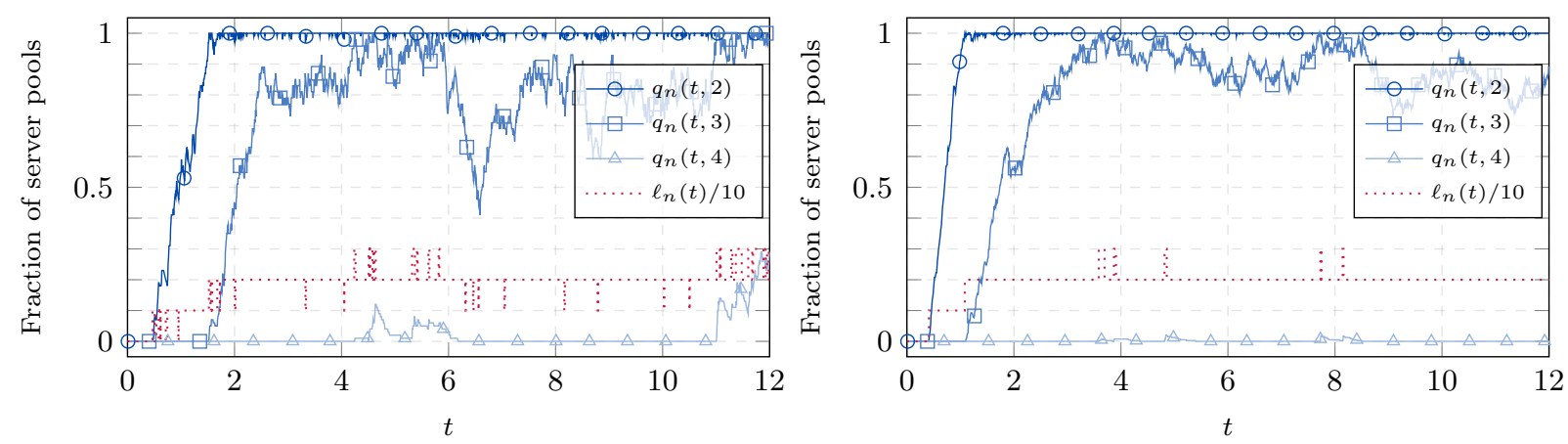

Figure 3: Evolution of $\ell_{n}$ over time when $\lambda=2.9$ and $\alpha=0.97$. On the left, $n=100$ and the threshold fluctuates between $\lfloor\lambda\rfloor-1$ and $\lceil\lambda\rceil$. On the right, $n=400$ and the threshold stays at $\lfloor\lambda\rfloor$ most of the time.

In the system with $n=400$ the threshold stays at $\lfloor\lambda\rfloor=2$ most of the time, with sporadic and brief excursions to $\lceil\lambda\rceil=3$. The oscillations disappear completely when $n=500$; this is not shown in Figure 3, but can be checked in the other experiments presented in this section, which correspond to $n=500$.

Besides the number of server pools, the convergence of $\ell_{n}$ depends on the fractional part of $\lambda$. Specifically, the larger $\lambda-\lfloor\lambda\rfloor$ is, the larger $n$ will have to be for $\ell_{n}$ to settle; observe that $\lambda-\lfloor\lambda\rfloor$ is relatively large in Figure 3. To understand this behavior, suppose $\ell_{n}$ has reached $\lfloor\lambda\rfloor$, then Theorem 6 suggests that $q_{n}(\lceil\lambda\rceil)$ will oscillate around $\lambda-\lfloor\lambda\rfloor$ with deviations of order $1 / \sqrt{n}$. If $\lambda-\lfloor\lambda\rfloor$ is large, then $n$ must also be large in order to prevent $q_{n}(\lceil\lambda\rceil)$ from reaching one with high probability.

We proceed to assess the upper bound (15) for the time until the threshold settles. Figure 4 shows trajectories of two systems with $\lambda=5.5, n=500$ and different initial conditions; in both cases $\alpha=0.93$ satisfies (14). While in the initially empty system $\ell_{n}$ settles at $\lfloor\lambda\rfloor=5$ almost exactly at $\bar{t}_{\text {eq }}$, in the initially overloaded system $\ell_{n}$ settles several units of time before $\bar{t}_{\text {eq }}$. In both cases the threshold settles quickly in less than the average time needed to execute three tasks. In Figure 4 we can also see the trajectories of $q_{n}(\lfloor\lambda\rfloor)$ and $q_{n}(\lceil\lambda\rceil)$ after the threshold has settled at the optimal value. The former trajectories present very subtle oscillations, whereas the latter exhibit more variability. This coincides with Theorem 6, which suggests that the deviations of $q_{n}(\lfloor\lambda\rfloor)$ from one are of order $\log (n) / n$ and that those of $q_{n}(\lceil\lambda\rceil)$ from $\lambda-\lfloor\lambda\rfloor$ are of order $1 / \sqrt{n}$.

We now evaluate the quality-of-service experienced by users. To this end we ran long simulations for several dispatching policies in stationarity, and we computed the empirical distribution of the fraction of resources received by an arbitrary user. We assumed the resources of any given server pool were equitably distributed among the tasks sharing it and we computed at each instant of time the number of tasks receiving a certain fraction of resources. We then integrated these quantities over time and normalized them to add up to one, as shown in Figure 5. The policy that assigns tasks to server pools chosen uniformly at random exhibits the largest variance, with some tasks receiving all the resources of a given server pool and some others contending for resources with as many as 15 tasks. 

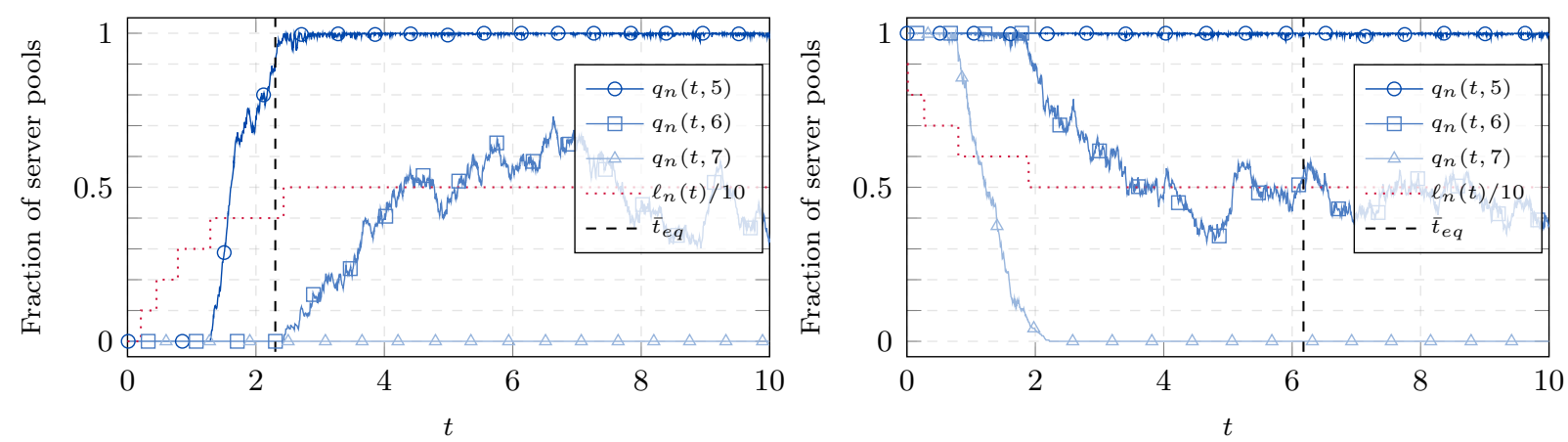

Figure 4: Time until the threshold settles when $\lambda=5.5, \alpha=0.93$ and $n=500$. The system on the left is initially empty and (15) is tight. On the right, all server pools have 9 tasks initially and (15) is not tight.

Users are treated more fairly when tasks are sent to the least congested of two server pools chosen uniformly at random, but still some tasks share a server pool with as many as 13 other tasks. Finally, the fraction of resources assigned to tasks is either $1 / 10$ or $1 / 11$ for virtually all tasks when the threshold policy or JSQ are used. For these two policies the load is evenly distributed and tasks are thus treated fairly, no users experience an inferior quality-of-service. In particular, the Schur-concave utilities mentioned in the Introduction, which measure the overall experienced performance, are maximal.

We conclude by investigating the response of the threshold policy to highly variable demand patterns. The trajectories depicted in Figure 6 correspond to a system where $\lambda$ is time-varying and $\alpha$ was chosen according to (13) for $\lambda_{\max }=10$. We observe that the system copes effectively with drastic and abrupt load variations, as the ones occurring around $t=0,20,30$; in all these cases $\ell_{n}$ quickly reaches the new optimal value. Also, the small but swift load fluctuations within the interval $[2,15]$ do not move the threshold away from the optimal value, which remains constant along the entire interval. Finally, $\ell_{n}$ adjusts to the slow load variations within $[33,45]$, which in this case result in changes of the optimal value. Along with the threshold, $m(t)=\max \left\{i: q_{n}(t, i)>0\right\}$ and the quadratic error $e(t)=\left\|q_{n}(t)-q^{*}(t)\right\|_{2}$ are plotted; here $q^{*}(t)$ is computed in terms of $\lambda(t)$ from (1). We observe that $m(t)=\lceil\lambda(t)\rceil$ most of the time and $e(t)$ is generally small, with its peak values coinciding with the most drastic changes of $\lambda(t)$; i.e., concentrations of tasks at individual server pools are avoided and the loads are close to balanced most of the time.

\section{Conclusions.}

In this paper we examined a self-learning threshold-based policy for dispatching tasks in a system of parallel server pools, with the aim of balancing the total number of tasks evenly. We proved that this policy achieves the latter objective asymptotically, both on the fluid and the diffusion scale. These results were supported by several numerical experiments which showed that the optimality properties can already be seen in systems with a few hundred server pools, and we presented additional simulations which indicate that our 


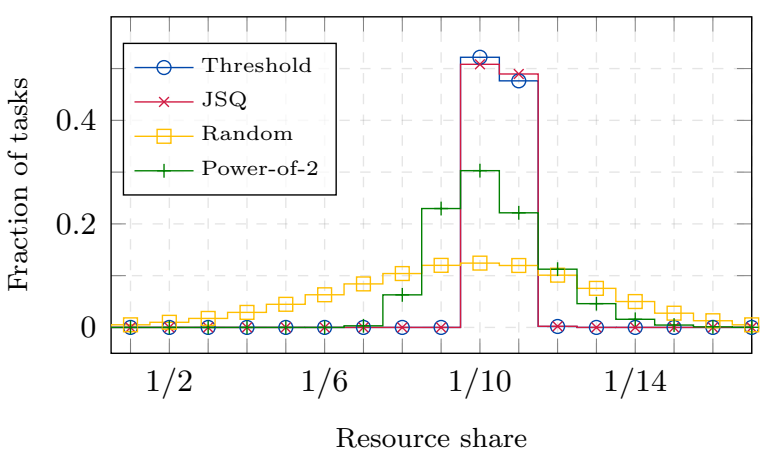

Figure 5: User-perceived performance under different dispatching rules. All policies were simulated with $\lambda=10.5$ and $n=500$.

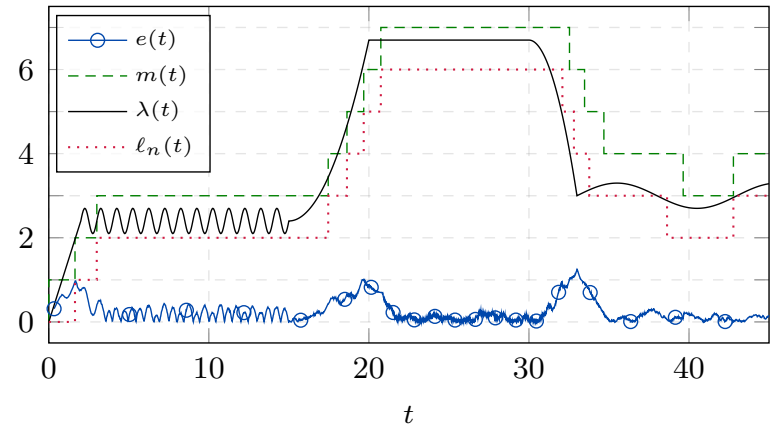

Figure 6: Response of the threshold policy to a highly variable demand pattern, with $n=500$ and $\alpha=0.91$ chosen from (13) for $\lambda_{\max }=10$.

policy copes effectively with highly variable demand patterns. Also, we provided a tokenbased implementation that involves at most two messages per task and storage of only little state information at the dispatcher, in the form of two tokens per server pool.

Our self-learning scheme adjusts the threshold over time to find an optimal value and, when an update occurs, the new threshold must be known by the dispatcher and all server pools. Synchronizing the threshold value of these entities could introduce a considerable communication burden during threshold updates. Although these updates occur only a few times before the threshold settles, a distributed procedure for adjusting the threshold could reduce the communication burden and the design of such a procedure is a topic for future research. Another possible line of future inquiry is to study whether our adaptive load balancing policy can be combined with mechanisms for adjusting the number of server pools over time, particularly in cloud computing deployments where the processing capacity is elastic. Specifically, the overall quality-of-service can be unacceptable if the offered load is too large, even if tasks are evenly distributed. In order to overcome this situation the capacity of the system could be increased by deploying more server pools.

Throughout the paper we considered systems of infinite server pools. Our self-learning threshold-based policy can be extended to scenarios where the maximum number of flows in each of the server pools is subject to a finite upper bound $B$, by imposing that the threshold cannot be increased beyond $B-1$. Unlike the infinite-server setting, this model allows to consider heavy-traffic regimes where the difference between total number of servers $n B$ and the arrival rate $\lambda_{n}$ of the $n^{\text {th }}$ system grows sublinearly with $n$. A topic of future research is the blocking probability of our threshold policy in these regimes, particularly in the Halfin-Whitt regime where $\left(\lambda_{n}-n B\right) / \sqrt{n}$ has a finite limit as $n$ grows to infinity.

Additional directions of future research concern extensions of our model to systems of heterogeneous server pools. One source of heterogeneity could be the way in which the service rate of tasks depends on the number of concurrent tasks at each server pool; in our model the service rate is equal to the number of tasks. The fluid limits derived in this paper carry over to the general setting, but establishing that the learning rule for adjusting the 
threshold always reaches an equilibrium requires non-trivial additional arguments since our proof relies on the property of infinite-server systems that the total number of tasks is not affected by the assignment decisions. Another source of heterogeneity could be the utility function $u$ mentioned in Section 2.1 as a proxy for measuring quality-of-service. It would be interesting to investigate how the results in this paper generalize to systems where the experienced performance depends on the type of server pool, and thus evenly balancing the load may not maximize the overall quality-of-service.

\section{Appendix A Proof of the fluid limits}

In this appendix we prove Theorems 1 and 9. In Section A.1 we define the sample paths of the discrete systems $s_{n}=\left(q_{n}, \ell_{n}\right)$ on a common probability space. In Section A.2 we prove that $\left\{\left.q_{n}\right|_{[0, T]}: n \geq 1\right\}$ is almost surely relatively compact, with respect to a suitable metric. In Section A.3 we assume that all systems have the same constant threshold and we complete the proof of Theorem 1. In Section A.4 the thresholds evolve as in Section 4 and we prove Theorem 9 .

\section{A.1 Construction on a common probability space}

Consider the following stochastic processes and random variables.

- Driving Poisson processes: a Poisson process $\mathcal{N}_{\lambda}$ of rate $\lambda$, for arrivals, and a sequence of Poisson processes $\left\{\mathcal{N}_{1}^{i}: i \geq 1\right\}$ of unit rates, for potential departures. These are independent processes defined on a common probability space $\left(\Omega_{D}, \mathcal{F}_{D}, \mathbb{P}_{D}\right)$.

- Selection variables: a sequence $\left\{U_{j}: j \geq 1\right\}$ of independent uniform random variables with values on $[0,1)$, defined on a common probability space $\left(\Omega_{S}, \mathcal{F}_{S}, \mathbb{P}_{S}\right)$.

- Initial conditions: two sequences, $\left\{q_{n}(0): n \geq 1\right\}$ and $\left\{\ell_{n}(0): n \geq 1\right\}$, of random elements describing the initial conditions of the systems. The random elements in the first of these sequences take values in $\mathcal{Q}_{n}=\{q \in Q: n q(i) \in \mathbb{N}$ for all $i \geq 0\}$ and the random variables in the second sequence take values in $\mathbb{N}$. All these random elements are defined on $\left(\Omega_{I}, \mathcal{F}_{I}, \mathbb{P}_{I}\right)$

For the last set of random variables, recall that

$$
Q=\left\{q \in[0,1]^{\mathbb{N}}: q(i+1) \leq q(i) \leq q(0)=1 \text { for all } i \geq 1\right\}
$$

The discrete systems $s_{n}=\left(q_{n}, \ell_{n}\right)$ will be defined on the product probability space $(\Omega, \mathcal{F}, \mathbb{P})$ of the spaces $\left(\Omega_{D}, \mathcal{F}_{D}, \mathbb{P}_{D}\right),\left(\Omega_{S}, \mathcal{F}_{S}, \mathbb{P}_{S}\right)$ and $\left(\Omega_{I}, \mathcal{F}_{I}, \mathbb{P}_{I}\right)$ from a set of implicit equations involving the latter stochastic primitives. This construction is standard; see $[28,29]$. Before writing these equations it is convenient to introduce some notation. 


\section{A.1.1 Preliminary notation}

Let $q \in Q$ represent the occupancy state of a system. The intervals

$$
I_{i}(q)=[1-q(i-1), 1-q(i)), \quad \text { with } \quad i \geq 1,
$$

form a partition of $[0,1)$ and have lengths which are proportional to the number of server pools with exactly $i-1$ tasks. In addition, if $q(\ell)<1$ for some $\ell \in \mathbb{N}$, representing the threshold, then we may also partition $[0,1)$ into the intervals

$$
J_{i}(q, \ell)=\left[\frac{1-q(i-1)}{1-q(\ell)}, \frac{1-q(i)}{1-q(\ell)}\right) \quad \text { with } \quad 1 \leq i \leq \ell .
$$

Observe that the length of $J_{i}(q, \ell)$ is the fraction of server pools with precisely $i-1$ tasks divided by the fraction of server pools with at most $\ell$ tasks; if $q(\ell)=1$ we adopt the convention $J_{i}(q, \ell)=\emptyset$ for all $1 \leq i \leq \ell$. Letting $h=\ell+1$ we may now define

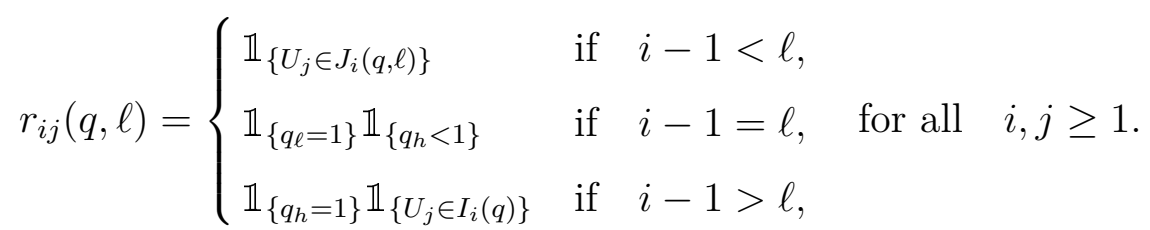

We will use these functions to take the load balancing decisions in the discrete systems. Specifically, if $q$ is the occupancy state and $\ell$ is the threshold, then the $j^{\text {th }}$ incoming task will be dispatched to a server pool with exactly $i-1$ tasks if and only if $r_{i j}(q, \ell)=1$. Observe that for each fixed $j$ the functions $r_{i j}(q, \ell)$ take values in $\{0,1\}$ and add up to one.

\section{A.1.2 Implicit equations}

For each pair of functions $q:[0, \infty) \longrightarrow Q$ and $\ell:[0, \infty) \longrightarrow \mathbb{N}$ we define two processes, $\mathcal{A}_{n}(q, \ell)$ and $\mathcal{D}_{n}(q)$, with values in $\mathbb{N}^{\mathbb{N}} / n$. The zero coordinates of these processes are identically zero. In order to define the other coordinates, let $\left\{\sigma_{n j}: j \geq 1\right\}$ denote the jump times of the Poisson process $\mathcal{N}_{\lambda}(n \cdot)$. We define:

$$
\begin{aligned}
& \mathcal{A}_{n}(q, \ell, t, i)=\frac{1}{n} \sum_{j=1}^{\mathcal{N}_{\lambda}(n t)} r_{i j}\left(q\left(\sigma_{n j}^{-}\right), \ell\left(\sigma_{n j}^{-}\right)\right), \\
& \mathcal{D}_{n}(q, t, i)=\frac{1}{n} \mathcal{N}_{1}^{i}\left(n \int_{0}^{t} i\left[q_{i}(s)-q_{i+1}(s)\right] d s\right) .
\end{aligned}
$$

Suppose the thresholds evolve according to the control rule described in Section 4, then the discrete system $s_{n}=\left(q_{n}, \ell_{n}\right)$ is defined as the unique solution to the following set of 
implicit equations:

$$
\begin{aligned}
& q(t)=q_{n}(0)+\mathcal{A}_{n}(q, \ell, t)-\mathcal{D}_{n}(q, t), \\
& \ell(t)=\ell_{n}(0)+\sum_{j=1}^{\mathcal{N}_{\lambda}(n t)}\left[\mathbb{1}_{\left\{q\left(\sigma_{n j}^{-}, h\left(\sigma_{n j}^{-}\right)\right) \geq 1-1 / n\right\}}-\mathbb{1}_{\left\{q\left(\sigma_{n j}^{-}, \ell\left(\sigma_{n j}^{-}\right)\right) \leq \alpha\right\}}\right],
\end{aligned}
$$

where $h(t)=\ell(t)+1$. If the thresholds are constant over time, then (17b) is replaced by $\ell_{n} \equiv \ell$ for some fixed threshold $\ell \in \mathbb{N}$. In both cases it is possible to prove, by induction on the jump times of the Poisson processes, that a solution defined on $[0, \infty)$ exists and is unique with probability one ${ }^{3}$. If we define $\mathcal{A}_{n}=\mathcal{A}_{n}\left(q_{n}, \ell_{n}\right)$ and $\mathcal{D}_{n}=\mathcal{D}_{n}\left(q_{n}, \ell_{n}\right)$, then we may interpret (17) as follows.

- The process $\mathcal{A}_{n}(i)$ counts the number of arrivals to server pools with exactly $i-1$ tasks. Whether it has a jump at an arrival epoch depends on the routing decisions encoded in the processes $r_{i j}\left(q_{n}, \ell_{n}\right)$. Observe that the random variables $\mathcal{A}_{n}(t, i)$ add up to $\mathcal{N}_{\lambda}(n t)$.

- The process $\mathcal{D}_{n}(i)$ counts the number of departures from server pools with exactly $i$ tasks. It is a Poisson process with time-varying intensity $n i\left[q_{n}(i)-q_{n}(i+1)\right]$, equal to the number of tasks in server pools with exactly $i$ tasks.

- At each arrival epoch $\ell_{n}$ is increased by one if and only if $n q_{n}\left(h_{n}\right) \geq n-1$ right before the arrival, and it is decreased by one if and only if $q_{n}\left(\ell_{n}\right) \leq \alpha$ right before the arrival.

Concluding, we note that the processes $\mathcal{A}_{n}$ and $\mathcal{D}_{n}$ have non-decreasing components which are equal to zero at time zero, and that $q_{n}$ takes values in $Q$ for all $n \geq 1$.

\section{A.2 Relative compactness of sample paths}

In this section we fix a finite time horizon $T$ and we prove that there exists a set of probability one where the restrictions to $[0, T]$ of the sample paths $\mathcal{A}_{n}(\omega), \mathcal{D}_{n}(\omega)$ and $q_{n}(\omega)$ form three relatively compact sequences such that the limit of each convergent subsequence is componentwise Lipschitz. For this purpose we must first specify a topology on the space of sample paths. With this in mind, we introduce the metric $d$ defined on $\mathbb{R}^{\mathbb{N}} \times \mathbb{R}^{\mathbb{N}}$ as

$$
d(a, b)=\sum_{i=0}^{\infty} \frac{\min \{|a(i)-b(i)|, 1\}}{2^{i}}
$$

\footnotetext{
${ }^{3}$ Strictly speaking, mild conditions have to be imposed on the random variables $q_{n}(0)$ in order to ensure that solutions do not explode in finite time. For instance, it is sufficient to assume that the coordinates of $q_{n}(0)$ have a finite sum with probability one; i.e., that the initial number of tasks in the system is finite with probability one.
} 
which corresponds to the product topology. We also consider the space $D_{\mathbb{R}^{\mathbb{N}}}[0, T]$ of all càdlàg functions on $[0, T]$ with values in $\mathbb{R}^{\mathbb{N}}$, endowed with the metric

$$
\rho_{u}^{\infty}(x, y)=\sup _{t \in[0, T]} d(x(t), y(t)) \quad \text { for all } \quad x, y \in D_{\mathbb{R}^{\mathbb{N}}}[0, T]
$$

We proceed under the following assumption regarding the initial occupancy states $q_{n}(0)$.

Assumption 1. There exists a random element $q(0)$, with values in $Q$, such that

$$
\lim _{n \rightarrow \infty} d\left(q_{n}(0), q(0)\right)=0 \quad \text { with probability one. }
$$

The subsequent arguments are based on [6]; see [39, Section 3.3.2] as well. These arguments make no use of the specific dynamics of the threshold. In particular, the results of this section hold both when the threshold is constant and when it is adjusted over time as in Section 4.

Proposition 17. There exists a set of probability one $\Gamma_{T}$ where the next conditions hold.

$$
\begin{aligned}
& \lim _{n \rightarrow \infty} d\left(q_{n}(0), q(0)\right)=0, \\
& \lim _{n \rightarrow \infty} \sup _{t \in[0, T]}\left|\frac{1}{n} \mathcal{N}_{\lambda}(n t)-\lambda t\right|=0, \\
& \lim _{n \rightarrow \infty} \sup _{t \in[0, i T]}\left|\frac{1}{n} \mathcal{N}_{1}^{i}(n t)-t\right|=0 \text { for all } i \geq 1 . \\
& \lim _{k \rightarrow \infty} \frac{1}{k} \sum_{j=1}^{k} \mathbb{1}_{\left\{U_{j} \in[a, b)\right\}}=b-a \text { for all }[a, b) \subset[0,1) .
\end{aligned}
$$

Proof. This result is a straightforward consequence of the functional strong law of large numbers for the Poisson process and the Glivenko-Cantelli theorem.

Remark 3. We will use equations (18a), (18b) and (18c) in this section, but we will not need (18d) here. This equation will be used later, to characterize the limit of convergent subsequences.

Note that (17a) and (18a) imply that we only need to prove that $\mathcal{A}_{n}(\omega)$ and $\mathcal{D}_{n}(\omega)$ form relatively compact sequences for all $\omega \in \Gamma_{T}$ and that the limit of each convergent subsequence has Lipschitz continuous coordinates. With this in mind, let us consider the space $D[0, T]$ of real càdlàg functions defined on $[0, T]$, in this case with the uniform metric:

$$
\rho_{u}(x, y)=\sup _{t \in[0, T]}|x(t)-y(t)| \quad \text { for all } \quad x, y \in D[0, T]
$$

Observe that a sequence $x_{n} \in D_{\mathbb{R}^{\mathbb{N}}}[0, T]$ converges to $x \in D_{\mathbb{R}^{\mathbb{N}}}[0, T]$ if and only if $x_{n}(i)$ 
converges to $x(i)$ with respect to $\rho_{u}$ for all $i \geq 0$. Furthermore, we have the following proposition.

Proposition 18. A sequence $\left\{x_{n}: n \geq 1\right\} \subset D_{\mathbb{R}^{\mathbb{N}}}[0, T]$ is relatively compact with respect to $\rho_{u}^{\infty}$ if and only if $\left\{x_{n}(i): n \geq 1\right\} \subset D[0, T]$ is relatively compact with respect to $\rho_{u}$ for all $i \geq 0$.

Proof. We only need to prove the converse, so let us assume the sequences $\left\{x_{n}(i): n \geq 1\right\}$ are relatively compact for all $i \geq 0$. Given an increasing sequence $\mathcal{K} \subset \mathbb{N}$, we must show that there exists a subsequence of $\left\{x_{k}: k \in \mathcal{K}\right\}$ that converges in $D_{\mathbb{R}^{\mathbb{N}}}[0, T]$. For this purpose we may construct a family of increasing sequences $\left\{\mathcal{J}_{i}: i \geq 0\right\}$ with the following properties.

(i) $\mathcal{J}_{i+1} \subset \mathcal{J}_{i} \subset \mathcal{K}$ for all $i \geq 0$.

(ii) $\left\{x_{j}(i): j \in \mathcal{J}_{i}\right\}$ has a limit $x(i) \in D[0, T]$ for each $i \geq 0$.

Consider the sequence $\left\{n_{j}: j \geq 1\right\} \subset \mathcal{K}$ such that $n_{j}$ is the $j^{\text {th }}$ element of $\mathcal{J}_{j}$, and note that

$$
\lim _{j \rightarrow \infty} \rho_{u}\left(x_{n_{j}}(i), x(i)\right)=0 \quad \text { for all } \quad i \geq 0 .
$$

Define $x \in D_{\mathbb{R}^{\mathbb{N}}}[0, T]$ so that its coordinates are the functions $x(i)$ introduced in (ii). Then $x_{n_{j}}$ converges to $x$ with respect to $\rho_{u}^{\infty}$, which completes the proof.

As a consequence of Proposition 18, it suffices to prove that $\mathcal{A}_{n}(\omega, i)$ and $\mathcal{D}_{n}(\omega, i)$ form relatively compact sequences for all $\omega \in \Gamma_{T}$ and all $i \geq 1$, and that the limit of each convergent subsequence is Lipschitz. To prove this we introduce the sets $E_{M}$ of all Lipschitz functions $x \in D[0, T]$ with constant $M>0$ and such that $x(0)=0$. These are compact subsets of $D[0, T]$ by the Arzelá-Ascoli theorem. For each $M>0$ and $\varepsilon>0$ we also consider the set $E_{M}(\varepsilon)$ of all $x \in D[0, T]$ such that $x(0)=0$ and

$$
|x(t)-x(s)| \leq M|t-s|+\varepsilon \quad \text { for all } \quad s, t \in[0, T]
$$

The following lemma is just a restatement of [6, Lemma 4.2].

Lemma 19. If $x \in E_{M}(\varepsilon)$, then there exists $y \in E_{M}$ such that $\rho_{u}(x, y) \leq 4 \varepsilon$.

Now we will proceed as follows. First, we will provide for each $\omega \in \Gamma_{T}$ and each $i \geq 1$ a sequence of positive numbers $\left\{\varepsilon_{i n}: n \geq 1\right\}$ converging to zero and such that

$$
\mathcal{A}_{n}(\omega, i) \in E_{\lambda}\left(\varepsilon_{i n}\right) \quad \text { and } \quad \mathcal{D}_{n}(\omega, i) \in E_{i}\left(\varepsilon_{i n}\right) \text { for all } n \geq 1
$$

Next, we will show that Lemma 19 and the compactness of the sets $E_{\lambda}$ and $E_{i}$ imply that each subsequence of $\left\{\mathcal{A}_{n}(\omega, i): n \geq 1\right\}$ and $\left\{\mathcal{D}_{n}(\omega, i): n \geq 1\right\}$ has a further subsequence which converges to a Lipschitz function. 
Lemma 20. For each $i \geq 1$ and each $\omega \in \Gamma_{T}$ there exist positive real numbers $\left\{\varepsilon_{\text {in }}: n \geq 1\right\}$ converging to zero and such that $\mathcal{A}_{n}(\omega, i) \in E_{\lambda}\left(\varepsilon_{i n}\right)$ and $\mathcal{D}_{n}(\omega, i) \in E_{i}\left(\varepsilon_{i n}\right)$ for all $n \geq 1$.

Proof. Fix $i \geq 1$ and $\omega \in \Gamma_{T}$, we will omit $\omega$ from the notation. For all $s, t \in[0, T]$ we have

$$
\left|\mathcal{A}_{n}(t, i)-\mathcal{A}_{n}(s, i)\right| \leq \frac{1}{n}\left|\mathcal{N}_{\lambda}(n t)-\mathcal{N}_{\lambda}(n s)\right| \leq \lambda|t-s|+2 \sup _{u \in[0, T]}\left|\frac{1}{n} \mathcal{N}_{\lambda}(n u)-\lambda u\right|
$$

By Proposition 17 we conclude that there exist positive numbers $\left\{\varepsilon_{n}^{1}: n \geq 1\right\}$ such that

$$
\left|\mathcal{A}_{n}(t, i)-\mathcal{A}_{n}(s, i)\right| \leq \lambda|t-s|+\varepsilon_{n}^{1} \quad \text { for all } \quad s, t \in[0, T] \quad \text { and } \quad \lim _{n \rightarrow \infty} \varepsilon_{n}^{1}=0 .
$$

For each $t \in[0, T]$ let us define

$$
J_{n}(t, i)=\int_{0}^{t} i\left[q_{n}(s, i)-q_{n}(s, i+1)\right] d s
$$

which has the following two properties:

$$
J_{n}(t, i) \leq i T \quad \text { and } \quad\left|J_{n}(t, i)-J_{n}(s, i)\right| \leq i|t-s| \quad \text { for all } \quad s, t \in[0, T] .
$$

Using these properties we obtain:

$$
\begin{aligned}
\left|\mathcal{D}_{n}(t, i)-\mathcal{D}_{n}(s, i)\right| & \leq \frac{1}{n}\left|\mathcal{N}_{1}^{i}\left(n J_{n}(t, i)\right)-\mathcal{N}_{1}^{i}\left(n J_{n}(s, i)\right)\right| \\
& \leq\left|J_{n}(t, i)-J_{n}(s, i)\right|+2 \sup _{u \in[0, T]}\left|\frac{1}{n} \mathcal{N}_{1}^{i}\left(n J_{n}(u, i)\right)-J_{n}(u, i)\right| \\
& \leq i|t-s|+2 \sup _{u \in[0, i T]}\left|\frac{1}{n} \mathcal{N}_{1}^{i}(n u)-u\right| \quad \text { for all } \quad s, t \in[0, T] .
\end{aligned}
$$

By (18c) there exist positive numbers $\left\{\varepsilon_{i n}^{2}: n \geq 1\right\}$ such that

$$
\left|\mathcal{D}_{n}(t, i)-\mathcal{D}_{n}(s, i)\right| \leq i|t-s|+\varepsilon_{i n}^{2} \quad \text { for all } \quad s, t \in[0, T] \text { and } \quad \lim _{n \rightarrow \infty} \varepsilon_{i n}^{2}=0
$$

The result follows letting $\varepsilon_{i n}=\varepsilon_{n}^{1}+\varepsilon_{i n}^{2}$.

Theorem 21. The restrictions to $[0, T]$ of the sample paths $\mathcal{A}_{n}(\omega), \mathcal{D}_{n}(\omega)$ and $q_{n}(\omega)$ form relatively compact sequences with respect to $\rho_{u}^{\infty}$ for all $\omega \in \Gamma_{T}$. Moreover, the limit of each convergent subsequence has Lipschitz coordinates.

Proof. Fix an arbitrary $\omega \in \Gamma_{T}$. As observed before, it is enough to show that for each $i \geq 1$ every subsequence of $\left\{\mathcal{A}_{n}(i): n \geq 1\right\}$ and $\left\{\mathcal{D}_{n}(i): n \geq 1\right\}$ has a further subsequence that converges with respect to $\rho_{u}$ to a Lipschitz continuous function.

We will prove this for $\left\{\mathcal{A}_{n}(i): n \geq 1\right\}$; the proof is similar for $\left\{\mathcal{D}_{n}(i): n \geq 1\right\}$. Fix $i \geq 1$, by Lemma 20 there exist $M_{i}$ and a sequence of positive numbers $\left\{\varepsilon_{i n}: n \geq 1\right\}$ such 
that

$$
\mathcal{A}_{n}(i) \in E_{M_{i}}\left(\varepsilon_{i n}\right) \quad \text { for all } n \geq 1 \quad \text { and } \quad \lim _{n \rightarrow \infty} \varepsilon_{i n}=0
$$

For each $n$ there exists $x_{n}(i) \in E_{M_{i}}$ such that $\rho_{u}\left(\mathcal{A}_{n}(i), x_{n}(i)\right) \leq 4 \varepsilon_{i n}$, by Lemma 19. In addition, the compactness of $E_{M_{i}}$ implies that every increasing sequence has a subsequence $\mathcal{K}$ such that $\left\{x_{k}(i): k \in \mathcal{K}\right\}$ converges to some $a(i) \in E_{M_{i}}$, and

$$
\lim _{k \rightarrow \infty} \rho_{u}\left(\mathcal{A}_{k}(i), a(i)\right) \leq \lim _{k \rightarrow \infty}\left[\rho_{u}\left(\mathcal{A}_{k}(i), x_{k}(i)\right)+\rho_{u}\left(x_{k}(i), a(i)\right)\right]=0
$$

This completes the proof.

Remark 4. As observed above, the proofs of the results stated in this section made no use of the specific dynamics of the threshold. In particular, the previous theorem holds regardless of how the threshold evolves over time.

\section{A.3 Systems with a static threshold}

In this section we consider systems with a constant threshold and we prove Theorem 1. Specifically, we assume that there exists $\ell \in \mathbb{N}$ such that $\ell_{n}(\omega, t)=\ell$ for all $n \geq 1$, all $\omega \in \Omega$ and all $t \geq 0$. We have shown that the restrictions to $[0, T]$ of the sample paths $q_{n}(\omega)$ form a relatively compact sequence for each $\omega \in \Gamma_{T}$, and below we characterize the limits of convergent subsequences.

For this purpose we fix some $\omega \in \Gamma_{T}$, which we omit from the notation, and an increasing sequence of natural numbers. By Theorem 21 there exist $a, d \in D_{\mathbb{R}^{\mathbb{N}}}[0, T]$ and a subsequence $\mathcal{K}$, of the aforementioned sequence, such that the following properties hold.

- $a(0)$ is identically zero, $a(i) \in E_{\lambda}$ for all $i \geq 1$ and

$$
\lim _{k \rightarrow \infty} \sup _{t \in[0, T]}\left|\mathcal{A}_{k}(t, i)-a(t, i)\right|=0 \quad \text { for all } \quad i \geq 1
$$

- $d(0)$ is identically zero, $d(i) \in E_{i}$ for all $i \geq 1$ and

$$
\lim _{k \rightarrow \infty} \sup _{t \in[0, T]}\left|\mathcal{D}_{k}(t, i)-d(t, i)\right|=0 \quad \text { for all } \quad i \geq 1
$$

In addition, note that $a(i)$ and $d(i)$ are non-negative and non-decreasing for all $i \geq 1$ since $\mathcal{A}_{n}(i)$ and $\mathcal{D}_{n}(i)$ have the same properties for all $n$ and all $i \geq 1$.

Consider the function $q \in D_{\mathbb{R}^{\mathbb{N}}}[0, T]$ defined by:

$$
q(t)=q(0)+a(t)-d(t) \quad \text { for all } \quad t \in[0, T]
$$


where $q(0)$ is as in Assumption 1. By (17a) and (18a) we know that

$$
\lim _{k \rightarrow \infty} \sup _{t \in[0, T]}\left|q_{k}(t, i)-q(t, i)\right|=0 \quad \text { for all } \quad i \geq 1
$$

this implies that $q$ takes values in $Q$. Since $a$ and $d$ have Lipschitz coordinates, there exists a set of total Lebesgue measure $\mathcal{R} \subset(0, T)$ where both $a(i)$ and $d(i)$ are differentiable for all $i \geq 1$. In the next lemma we compute the derivatives of $a$ and $d$ within this set of regular points. Theorem 1 is a straightforward consequence of Theorem 21 and this lemma.

Lemma 22. Given a regular point $t_{0} \in \mathcal{R}$, we have

$$
\dot{d}\left(t_{0}, i\right)=i\left[q\left(t_{0}, i\right)-q\left(t_{0}, i+1\right)\right] \quad \text { for all } i \geq 1 \text {. }
$$

As usual, let us define $h=\ell+1$. The derivatives $\dot{a}\left(t_{0}, i\right)$ are as follows.

(a) If $q\left(t_{0}, \ell\right)<1$, then

$$
\dot{a}\left(t_{0}, i\right)= \begin{cases}\lambda\left[\frac{q\left(t_{0}, i-1\right)-q\left(t_{0}, i\right)}{1-q\left(t_{0}, \ell\right)}\right] & \text { if } \quad 1 \leq i \leq \ell \\ 0 & \text { if } \quad i \geq h\end{cases}
$$

(b) If $q\left(t_{0}, \ell\right)=1$ and $q\left(t_{0}, h\right)<1$, then

$$
\dot{a}\left(t_{0}, i\right)= \begin{cases}\ell\left[1-q\left(t_{0}, h\right)\right] & \text { if } \quad i=\ell, \\ \lambda-\ell\left[1-q\left(t_{0}, h\right)\right] & \text { if } \quad i=h \\ 0 & \text { if } \quad i \neq \ell, h\end{cases}
$$

(c) If $q\left(t_{0}, h\right)=1$, then

$$
\dot{a}\left(t_{0}, i\right)= \begin{cases}h\left[1-q\left(t_{0}, h+1\right)\right] & \text { if } \quad i=h, \\ {\left[\lambda-h\left(1-q\left(t_{0}, h+1\right)\right)\right]\left[q\left(t_{0}, i-1\right)-q\left(t_{0}, i\right)\right]} & \text { if } i \geq h+1 \\ 0 & \text { if } \quad 1 \leq i \leq \ell .\end{cases}
$$

Proof. To compute $\dot{d}\left(t_{0}, i\right)$, it is enough to show that

$$
d(t, i)=\int_{0}^{t} i[q(s, i)-q(s, i+1)] d s \text { for all } i \geq 1 \text { and } t \geq 0 .
$$

To this purpose, we note that the difference between these two expressions is bounded in 
absolute value by the sum of the following three terms:

$$
\begin{aligned}
& \left|d(t, i)-\mathcal{D}_{k}(t, i)\right| \\
& \left|\mathcal{D}_{k}(t, i)-\int_{0}^{t} i\left[q_{k}(s, i)-q_{k}(s, i+1)\right] d s\right| \\
& \left|\int_{0}^{t} i\left[q_{k}(s, i)-q_{k}(s, i+1)\right] d s-\int_{0}^{t} i[q(s, i)-q(s, i+1)] d s\right| .
\end{aligned}
$$

Observe that as $k \rightarrow \infty$ each of these expressions converges to zero: the first one by (20), the second one by (18c) and the third one by (21). This establishes (22).

To prove (a) we fix $1 \leq i \leq \ell$ and $\varepsilon>0$. Note that $q_{k}(i-1), q_{k}(i)$ and $q_{k}(\ell)$ converge to the continuous functions $q(i-1), q(i)$ and $q(\ell)$, uniformly over $[0, T]$. So there exists $\delta>0$ such that

$$
\frac{1-q_{k}(t, i-1)}{1-q_{k}(t, \ell)} \geq \frac{1-q\left(t_{0}, i-1\right)}{1-q\left(t_{0}, \ell\right)}-\varepsilon \quad \text { and } \quad \frac{1-q_{k}(t, i)}{1-q_{k}(t, \ell)} \leq \frac{1-q\left(t_{0}, i\right)}{1-q\left(t_{0}, \ell\right)}+\varepsilon
$$

for all $t \in\left(t_{0}-\delta, t_{0}+\delta\right)$ and all large enough $k \in \mathcal{K}$. For such $t$ and $k$ we have

$$
\tilde{J}_{i}=\left[\frac{1-q\left(t_{0}, i-1\right)}{1-q\left(t_{0}, \ell\right)}-\varepsilon, \frac{1-q\left(t_{0}, i\right)}{1-q\left(t_{0}, \ell\right)}+\varepsilon\right) \supset J_{i}\left(q_{k}(t)\right) .
$$

Recalling the definition of $\mathcal{A}_{k}(i)$, we conclude that

$$
\begin{aligned}
\mathcal{A}_{k}(t, i)-\mathcal{A}_{k}\left(t_{0}, i\right) & =\frac{1}{k} \sum_{j=\mathcal{N}_{\lambda}\left(k t_{0}\right)+1}^{\mathcal{N}_{\lambda}(k t)} \mathbb{1}_{\left\{U_{j} \in J_{i}\left(q_{k}\left(\sigma_{k j}^{-}\right)\right)\right\}} \\
& \leq \frac{1}{k} \sum_{j=\mathcal{N}_{\lambda}\left(k t_{0}\right)+1}^{\mathcal{N}_{\lambda}(k t)} \mathbb{1}_{\left\{U_{j} \in \tilde{J}_{i}\right\}} \\
& =\frac{\mathcal{N}_{\lambda}(k t)}{k} \frac{1}{\mathcal{N}_{\lambda}(k t)} \sum_{j=1}^{\mathcal{N}_{\lambda}(k t)} \mathbb{1}_{\left\{U_{j} \in \tilde{J}_{i}\right\}}-\frac{\mathcal{N}_{\lambda}\left(k t_{0}\right)}{k} \frac{1}{\mathcal{N}_{\lambda}\left(k t_{0}\right)} \sum_{j=1}^{\mathcal{N}_{\lambda}\left(k t_{0}\right)} \mathbb{1}_{\left\{U_{j} \in \tilde{J}_{i}\right\}}
\end{aligned}
$$

for all $t \in\left(t_{0}-\delta, t_{0}+\delta\right)$ and all sufficiently large $k \in \mathcal{K}$. Taking the limit as $k \rightarrow \infty$ on the left-most and right-most sides, we obtain

$$
a(t, i)-a\left(t_{0}, i\right) \leq \lambda\left(t-t_{0}\right)\left[\frac{q\left(t_{0}, i-1\right)-q\left(t_{0}, i\right)}{1-q\left(t_{0}, \ell\right)}+2 \varepsilon\right]
$$

in order to compute the limit of the right-hand side we used (18b) and (18d). Note that (23) is preserved if $t \in\left(t_{0}, t_{0}+\delta\right)$ and we divide both sides by $t-t_{0}$. If we then take the limit as $t \rightarrow t_{0}^{+}$, and next we take the limit as $\varepsilon \rightarrow 0^{+}$, we get

$$
\dot{a}\left(t_{0}, i\right) \leq \lambda\left[\frac{q\left(t_{0}, i-1\right)-q\left(t_{0}, i\right)}{1-q\left(t_{0}, \ell\right)}\right] .
$$


If instead $t \in\left(t_{0}-\delta, t_{0}\right)$, then (23) is reversed when we divide by $t-t_{0}$. Taking in this case the limit as $t \rightarrow t_{0}^{-}$and then the limit as $\varepsilon \rightarrow 0^{+}$, we see that equality holds above, establishing (a).

Turning now to (b), we note that there exists $\varepsilon>0$ such that $q(t, h)<1$ for all $t \in\left(t_{0}-\varepsilon, t_{0}+\varepsilon\right)$. This is also true for $q_{k}(h)$ and all large enough $k \in \mathcal{K}$, which means that tasks arriving to large enough systems during $\left(t_{0}-\varepsilon, t_{0}+\varepsilon\right)$ are exclusively sent to server pools with at most $\ell$ tasks. Thus,

$$
\begin{aligned}
& \sum_{i=1}^{h}\left[\mathcal{A}_{k}(t, i)-\mathcal{A}_{k}\left(t_{0}, i\right)\right]=\frac{1}{k}\left[\mathcal{N}_{\lambda}(k t)-\mathcal{N}_{\lambda}\left(k t_{0}\right)\right], \\
& \mathcal{A}_{k}(t, i)=\mathcal{A}_{k}\left(t_{0}, i\right) \quad \text { for all } \quad i \geq h+1,
\end{aligned}
$$

for all $t \in\left(t_{0}-\varepsilon, t_{0}+\varepsilon\right)$ and all sufficiently large $k \in \mathcal{K}$. The right-hand side of the first equation converges uniformly over $\left(t_{0}-\varepsilon, t_{0}+\varepsilon\right)$ to $\lambda\left(t-t_{0}\right)$ by $(18 \mathrm{~b})$. By taking the limit as $k \rightarrow \infty$ on both sides of (24a), dividing both sides by $t-t_{0}$ next, and then taking the limit as $t \rightarrow t_{0}$, we get

$$
\sum_{i=1}^{h} \dot{a}\left(t_{0}, i\right)=\lambda
$$

Furthermore, we conclude from (24b) that

$$
\dot{a}\left(t_{0}, i\right)=0 \quad \text { for all } \quad i \geq h+1 \text {. }
$$

Now note that $q\left(t_{0}, i\right)=1$ implies

$$
0 \leq \lim _{t \rightarrow t_{0}^{-}} \frac{q(t, i)-q\left(t_{0}, i\right)}{t-t_{0}}=\dot{q}\left(t_{0}, i\right)=\lim _{t \rightarrow t_{0}^{+}} \frac{q(t, i)-q\left(t_{0}, i\right)}{t-t_{0}} \leq 0
$$

since $t_{0} \in(0, T)$ we may take both left and right limits. In particular, $q\left(t_{0}, \ell\right)=1$ implies $\dot{q}\left(t_{0}, i\right)=0$ for all $1 \leq i \leq \ell$. We conclude that

$$
\dot{a}\left(t_{0}, i\right)=\dot{d}\left(t_{0}, i\right)= \begin{cases}0 & \text { if } \quad 1 \leq i \leq \ell-1 \\ \ell\left[1-q\left(t_{0}, i\right)\right] & \text { if } \quad i=\ell .\end{cases}
$$

Finally, we obtain (b) using equations (25) and (26).

Suppose $q\left(t_{0}, h\right)=1$ to prove (c). As in the previous paragraph,

$$
\dot{a}\left(t_{0}, i\right)=\dot{d}\left(t_{0}, i\right)= \begin{cases}0 & \text { if } \quad 1 \leq i \leq \ell \\ h\left[1-q\left(t_{0}, h+1\right)\right] & \text { if } \quad i=h .\end{cases}
$$

To compute the other derivatives let us fix $i \geq h+1$, and recall that $\left\{\sigma_{k j}: j \geq 1\right\}$ are the 
jump times of $\mathcal{N}_{\lambda}(k \cdot)$. For each $t \geq t_{0}$ we define

$$
\mathcal{C}_{k}(t)=\frac{1}{k} \sum_{j=\mathcal{N}_{\lambda}\left(k t_{0}\right)+1}^{\mathcal{N}_{\lambda}(k t)} \mathbb{1}_{\left\{q_{k}\left(\sigma_{k j}^{-}, h\right)=1\right\}}=\frac{1}{k}\left[\mathcal{N}_{\lambda}(k t)-\mathcal{N}_{\lambda}\left(k t_{0}\right)\right]-\sum_{j=1}^{h}\left[\mathcal{A}_{k}(t, j)-\mathcal{A}_{k}\left(t_{0}, j\right)\right],
$$

and for each $t \in\left[0, t_{0}\right)$ we define $\mathcal{C}_{k}(t)$ by the expression after the first equality preceded by a minus sign. Observe that equations (18b) and (19) imply that

$$
\lim _{k \rightarrow \infty} \sup _{t \in[0, T]}\left|\mathcal{C}_{k}(t)-\left[\lambda\left(t-t_{0}\right)-\sum_{j=1}^{h}\left(a(t, j)-a\left(t_{0}, j\right)\right)\right]\right|=0
$$

Furthermore, if we let $\left\{\theta_{k j}: j \geq 1\right\}$ be the jump times of $\mathcal{C}_{k}$ then

$$
\mathcal{A}_{k}(t, i)-\mathcal{A}_{k}\left(t_{0}, i\right)=\frac{1}{k} \sum_{j=1}^{\mathcal{C}_{k}(t)} \mathbb{1}_{\left\{U_{j} \in I_{i}\left(q_{k}\left(\theta_{k j}^{-}\right)\right)\right\}} .
$$

Reasoning as in the proof of (a) we see that

$$
\dot{a}\left(t_{0}, i\right)=\left[\lambda-\sum_{j=1}^{h} \dot{a}\left(t_{0}, j\right)\right]\left[q\left(t_{0}, i-1\right)-q\left(t_{0}, i\right)\right]
$$

this equation is analogous to (23) after taking the limits with respect to $t$ and $\varepsilon$. Finally, (27) yields

$$
\dot{a}\left(t_{0}, i\right)=\left[\lambda-h\left(1-q\left(t_{0}, h+1\right)\right)\right]\left[q\left(t_{0}, i-1\right)-q\left(t_{0}, i\right)\right],
$$

which completes the proof of (c).

Remark 5. The previous lemma also holds if the threshold is only constant in a neighborhood of the regular point $t_{0}$. More precisely, if there exists $\varepsilon>0$ such that $\ell_{k}(t)=\ell$ for all $t \in\left(t_{0}-\varepsilon, t_{0}+\varepsilon\right)$ and all sufficiently large $k \in \mathcal{K}$. The proof only changes slightly.

\section{A.4 Systems with a dynamic threshold}

In this section we look into systems where the threshold changes over time according to the control rule described in Section 4 and we prove Theorem 9. More formally, we consider the restrictions to a finite interval of time $[0, T]$ of the discrete systems $s_{n}=\left(q_{n}, \ell_{n}\right)$. We prove that this sequence of functions is relatively compact with probability one, with respect to the metric $\varrho$ introduced in Section 4.3, and we characterize the limits of convergent subsequences. Recall from Assumption 1 that the initial occupancy states $q_{n}(0)$ converge to a random element $q(0)$ with probability one. We introduce a similar hypothesis regarding the initial thresholds. 
Assumption 2. There exists a random variable $\ell(0)$, taking values in $\mathbb{N}$, such that

$$
\lim _{n \rightarrow \infty} \ell_{n}(0)=\ell(0) \quad \text { with probability one. }
$$

Equivalently, there exists a set of probability one $\Gamma^{\ell}$ with the following property: for each $\omega \in \Gamma^{\ell}$ there exists $n(\omega)$ such that $\ell_{n}(\omega, 0)=\ell(\omega, 0)$ for all $n \geq n(\omega)$.

In order to prove Theorem 9 , we will approximate the discrete systems $s_{n}=\left(q_{n}, \ell_{n}\right)$ by systems where the threshold is updated only finitely many times. Specifically, the system with $n$ server pools where the threshold is updated only the first $m$ times will be denoted $s_{n}^{m}=\left(q_{n}^{m}, \ell_{n}^{m}\right)$. This system behaves exactly as $s_{n}$ until the threshold is updated for the last time.

More formally, let $\left\{\tau_{n}^{m}: m \geq 0\right\}$ be the threshold update times in $s_{n}$, under the convention that $\tau_{n}^{0}=0$. The system with $m$ updates may be constructed on $(\Omega, \mathcal{F}, \mathbb{P})$ as in Section A.1 from the initial value problem (17), but with (17b) replaced by

$$
\ell(t)=\ell_{n}(t) \mathbb{1}_{\left\{t<\tau_{n}^{m}\right\}}+\ell_{n}\left(\tau_{n}^{m}\right) \mathbb{1}_{\left\{t \geq \tau_{n}^{m}\right\}} .
$$

As in Section A.1, the initial value problem (17a)-(17b') has a unique solution $s_{n}^{m}=\left(q_{n}^{m}, \ell_{n}^{m}\right)$ defined on $[0, \infty)$ with probability one. Furthermore, we have

$$
q_{n}^{m}(t)=q_{n}(t) \quad \text { for all } \quad t \in\left[0, \tau_{n}^{m}\right]
$$

The sequence of sample paths $q_{n}^{m}(\omega) \in D_{\mathbb{R}^{\mathbb{N}}}[0, T+1]$ is relatively compact for each $\omega \in \Gamma_{T+1}$ as in Section A.2. The next proposition is a simple consequence of this observation, the proof is straightforward using a diagonal argument.

Proposition 23. Let us fix $\omega$ in the set of probability one $\Gamma_{T}^{\ell}=\Gamma_{T+1} \cap \Gamma^{\ell}$, then the following hold.

(a) $\ell_{n}(0)=\ell(0)$ for all sufficiently large $n$.

(b) Every increasing sequence has a subsequence $\mathcal{K}$ such that $\left\{q_{k}: k \in \mathcal{K}\right\}$ and $\left\{q_{k}^{m}: k \in \mathcal{K}\right\}$, for all $m \geq 1$, converge in $D_{\mathbb{R}^{\mathbb{N}}}[0, T+1]$.

Below we prove Theorem 9. Namely, we show that for each $\omega \in \Gamma_{T}^{\ell}$ every increasing sequence of natural numbers has a subsequence $\mathcal{K}$ such that $\left\{s_{k}(\omega): k \in \mathcal{K}\right\}$ converges with respect to $\varrho$, and that the limit $s(\omega) \in S$ may be extended to a fluid system. Recall from Definition 7 that the threshold of a fluid system maintains its initial value for a positive period of time. We introduce a technical hypothesis to ensure that the limits of discrete systems also have this property. 
Assumption 3. The limiting initial conditions $q(0)$ and $\ell(0)$ are such that

$$
q(\omega, 0, \ell(\omega, 0))>\alpha \quad \text { and } \quad q(\omega, 0, h(\omega, 0))<1 \quad \text { for all } \quad \omega \in \Gamma^{\ell}
$$

where $h=\ell+1$ as usual.

Suppose briefly that this assumption does not hold. For instance, assume $q(0, \ell(0)-j)<$ $\alpha$ had positive probability for some $j \geq 0$ and fix $\omega \in \Omega$ such that this condition holds. For this sample path $q_{n}(0, \ell(0)-j)<\alpha$ for all sufficiently large $n$, so the threshold decreases with each of the first $j+1$ arrivals, and this yields $\tau_{n}^{j+1} \rightarrow 0$ as $n \rightarrow \infty$. If the discrete systems $s_{n}$ had a convergent subsequence, then the initial threshold of the limit would be updated instantly, at least $j+1$ times, until it reached a value $\tilde{\ell}(0)$ such that $q(0, \tilde{\ell}(0))>\alpha$ as in Assumption 3. The purpose of the above assumption is to preclude these multiple threshold updates at time zero by assuming that the threshold already starts at the "right" value $\tilde{\ell}(0)$.

In order to demonstrate that the discrete systems $s_{n}$ have relatively compact sample paths, we will resort to an inductive argument using the systems $s_{n}^{m}$ where the threshold is updated only finitely many times. The induction step will be carried out in Lemma 25, which depends on the following property of the fluid dynamics (2).

Lemma 24. Consider a solution $q:[a, b] \longrightarrow Q$ of (2) for a threshold $\ell \in \mathbb{N}$, and suppose that $q(t, \ell) \geq \alpha$ for all $t \in[a, b)$ and $q(b, \ell)=\alpha$. Then $q(b, \ell-1)>\alpha$.

Proof. Suppose the claim is false: there exists $1 \leq i \leq \ell-1$ such that $q(b, \ell-i)=\alpha$. Let $m$ be the largest integer with this property and let

$$
f(t)=\lambda p_{\ell-m}(q(t), \ell)-(\ell-m)[q(t, \ell-m)-q(t, \ell-m+1)] \quad \text { for all } \quad t \in[a, b] .
$$

Note that $\dot{q}(\ell-m)=f$ almost everywhere on $[a, b]$. Also, $f$ is continuous in a left neighborhood of $b$ because $q$ has continuous coordinates and $q(b, \ell)<1$. Moreover, by the maximality of $m$,

$$
f(b)=\lambda\left[\frac{q(b, \ell-m-1)-q(b, \ell-m)}{1-q(b, \ell)}\right]>0 .
$$

By continuity, there exists an open left neighborhood of $b$ where $f \geq f(b) / 2$. As a result, since $q(t, \ell-m) \geq q(t, \ell) \geq \alpha$ for all $t \in[a, b)$, and $\dot{q}(t, \ell-m) \geq f(b) / 2>0$ almost everywhere in a left neighborhood of $\tau$, we conclude that $q(b, \ell-m)>\alpha$, a contradiction.

Lemma 25. For a fixed $\omega \in \Gamma_{T}^{\ell}$ suppose that there exist $m \geq 0$, an increasing sequence 
$\mathcal{K} \subset \mathbb{N}$, increasing times $\left\{\tau_{i}: 0 \leq i \leq m\right\}$ and thresholds $\left\{\ell_{i}: 0 \leq i \leq m\right\}$, such that

$$
\lim _{k \rightarrow \infty} \tau_{k}^{i}=\tau_{i} \quad \text { and } \quad \lim _{k \rightarrow \infty} \ell_{k}\left(\tau_{k}^{i}\right)=\ell_{i} \quad \text { for all } \quad 0 \leq i \leq m
$$

Suppose also $\left\{q_{k}^{m}: k \in \mathcal{K}\right\}$ and $\left\{q_{k}: k \in \mathcal{K}\right\}$ converge to $q^{m}, q \in D_{\mathbb{R}^{\mathbb{N}}}[0, T+1]$, respectively, and

$$
q\left(\tau_{m}, \ell_{m}\right)>\alpha \text { and } q\left(\tau_{m}, h_{m}\right)<1
$$

Here $h_{i}=\ell_{i}+1$, as usual. The following statements hold.

(a) For all $i \geq 1$, and almost everywhere on $\left[\tau_{m}, T+1\right]$, we have

$$
\dot{q}^{m}(i)=\lambda p_{i}\left(q^{m}, \ell_{m}\right)-i\left[q^{m}(i)-q^{m}(i+1)\right]
$$

(b) If there exists $\varepsilon \in(0,1)$ such that $q^{m}(t)=q(t)$ for all $t \in[0, T+\varepsilon]$, then

$$
\liminf _{k \rightarrow \infty} \tau_{k}^{m+1}>T
$$

(c) If for all $\varepsilon \in(0,1)$ there exists $t<T+\varepsilon$ such that $q^{m}(t) \neq q(t)$, then

$$
\tau_{m+1}=\inf \left\{t \geq \tau_{m}: q^{m}(t) \neq q(t)\right\} \in[0, T] \quad \text { and } \quad \lim _{k \rightarrow \infty} \tau_{k}^{m+1}=\tau_{m+1}
$$

In addition, either $q\left(\tau_{m+1}, \ell_{m}\right)=\alpha$ or $q\left(\tau_{m+1}, h_{m}\right)=1$, and

$$
\ell_{m+1}=\lim _{k \rightarrow \infty} \ell_{k}\left(\tau_{k}^{m+1}\right)=\left\{\begin{array}{lll}
\ell_{m}-1 & \text { if } & q\left(\tau_{m+1}, \ell_{m}\right)=\alpha \\
\ell_{m}+1 & \text { if } & q\left(\tau_{m+1}, h_{m}\right)=1
\end{array}\right.
$$

Moreover, the above constants, $\tau_{m+1}$ and $\ell_{m+1}$, satisfy:

$$
\tau_{m+1}>\tau_{m}, \quad q\left(\tau_{m+1}, \ell_{m+1}\right)>\alpha \quad \text { and } \quad q\left(\tau_{m+1}, h_{m+1}\right)<1 .
$$

Proof. In order to prove (a) we fix $t \in\left(\tau_{m}, T+1\right)$ such that the derivatives of all the coordinate functions $q^{m}(i)$ exist at time $t$; recall that these functions are Lipschitz continuous and thus almost everywhere differentiable. For all sufficiently large $k \in \mathcal{K}$ we have $\tau_{k}^{m}<t$ and $\ell_{k}=\ell_{m}$ in a neighborhood of $t$. Therefore, (a) follows from Remark 5 .

We now turn to the proof of (b). To prove this statement we will assume that there exists $\varepsilon \in(0,1)$ such that $q^{m}(t)=q(t)$ for all $t \in[0, T+\varepsilon]$, and that the limit inferior of $\tau_{k}^{m+1}$ is smaller than or equal to $T$. This implies there exists an increasing sequence $\mathcal{J} \subset \mathcal{K}$ such that

$$
\tau_{j}^{m+1} \leq T+\frac{\varepsilon}{2} \quad \text { for all } \quad j \in \mathcal{J} \quad \text { and } \quad \lim _{j \rightarrow \infty} \tau_{j}^{m+1}=\tilde{\tau}_{m+1}
$$


Below we will demonstrate that the latter assumption cannot hold by arriving to a contradiction and thus establishing (b). We start with the following two observations.

(i) First, $q\left(t, \ell_{m}\right) \geq \alpha$ for all $t \in\left[\tau_{m}, \tilde{\tau}_{m+1}\right]$. To understand why, fix $t \in\left(\tau_{m}, \tilde{\tau}_{m+1}\right)$ and pick, for each $j \in \mathcal{J}$, a jump time $\sigma_{j}$ of $\mathcal{N}_{\lambda}(j \cdot)$, so that $\sigma_{j} \rightarrow t$ as $j \rightarrow \infty$. For all sufficiently large $j$ we have $\sigma_{j} \in\left(\tau_{j}^{m}, \tau_{j}^{m+1}\right)$ and $\ell_{j}\left(\sigma_{j}\right)=\ell_{m}$, which implies that $q_{j}\left(\sigma_{j}, \ell_{m}\right)>\alpha$. We conclude that $q\left(t, \ell_{m}\right) \geq \alpha$. By continuity of $q$ this also holds at $t=\tau_{m}$ and $t=\tilde{\tau}_{m+1}$.

(ii) Second, $q\left(h_{m}+1\right)$ is a non-increasing function along the invterval $\left[\tau_{m}, \tilde{\tau}_{m+1}\right]$. Indeed, note that $h_{j}(t)=h_{m}$ for all $t \in\left[\tau_{j}^{m}, \tau_{j}^{m+1}\right)$ and all sufficiently large $j \in \mathcal{J}$, and observe that $q_{j}\left(h_{j}+1\right)$ does not increase along the interval $\left[\tau_{j}^{m}, \tau_{j}^{m+1}\right)$.

The threshold $\ell_{j}$ is updated at $\tau_{j}^{m+1}$, thus we also have $q\left(\tilde{\tau}_{m+1}, \ell_{m}\right)=\alpha$ or $q\left(\tilde{\tau}_{m+1}, h_{m}\right)=$ 1. Below we analyze these two situations separately, and in both cases we conclude that $q^{m}$ and $q$ cannot be identically equal in a right neighborhood of $\tilde{\tau}_{m+1}<T+\varepsilon$, which is the desired contradiction.

Suppose first that $q\left(\tilde{\tau}_{m+1}, \ell_{m}\right)=\alpha$, which corresponds to $\ell_{j}\left(\tau_{j}^{m+1}\right) \rightarrow \ell_{m}-1$ as $j \rightarrow \infty$; i.e., $\ell_{j}$ decreases at $\tau_{j}^{m+1}$ for all sufficiently large $j \in \mathcal{J}$. In this case, (a) and (i) imply that $q^{m}$ satisfies the hypotheses of Lemma 24 along $\left[\tau_{m}, \tilde{\tau}_{m+1}\right]$. As a result, $q\left(\tilde{\tau}_{m+1}, \ell_{m}-1\right)>\alpha$, so there exists $\delta>0$ such that $q_{j}\left(\tau_{j}^{m+1}, \ell_{m}-1\right) \geq \alpha+\delta$ for all sufficiently large $j \in \mathcal{J}$. This implies that

$$
\liminf _{j \rightarrow \infty}\left(\tau_{j}^{m+2}-\tau_{j}^{m+1}\right)>0
$$

Indeed, for all large enough $j$ the threshold adopted at $\tau_{j}^{m+1}$ is $\ell_{m}-1$. After this update, at least $\delta j$ tasks must leave before $q_{j}\left(\ell_{m}-1\right)$ reaches $\alpha$ and at least $(1-\alpha) j$ tasks must arrive before $q_{j}\left(\ell_{m}\right)$ reaches one. Hence, we conclude from (18b) and (18c) that

$$
\liminf _{j \rightarrow \infty}\left(\tau_{j}^{m+2}-\tau_{j}^{m+1}\right) \geq \min \left\{\delta, \frac{1-\alpha}{\lambda}\right\}>0
$$

Using Remark 5 we see that

$$
q(t, i)=q\left(\tilde{\tau}_{m+1}, i\right)+\int_{\tilde{\tau}_{m+1}}^{t} \lambda p_{i}\left(q(s), \ell_{m}-1\right) d s-\int_{\tilde{\tau}_{m+1}}^{t} i[q(s, i)-q(s, i+1)] d s
$$

for all $i \geq 1$ and all $t$ in a right neighborhood of $\tilde{\tau}_{m+1}$. Nevertheless, $q^{m}$ satisfies the same integral equations but with $\ell_{m}-1$ replaced by $\ell_{m}$, in the same right neighborhood of $\tilde{\tau}_{m+1}$. This leads to a contradiction, because we had assumed that $q^{m}(t)=q(t)$ in this neighborhood.

Suppose now that $q\left(\tilde{\tau}_{m+1}, h_{m}\right)=1$, which corresponds to $\ell_{j}\left(\tau_{j}^{m+1}\right) \rightarrow \ell_{m}+1$ as $j \rightarrow \infty$; i.e., $\ell_{j}$ increases at $\tau_{j}^{m+1}$ for all sufficiently large $j \in \mathcal{J}$. In this case, (30) and (ii) imply 
that

$$
q\left(\tilde{\tau}_{m+1}, h_{m}+1\right) \leq q\left(\tau_{m}, h_{m}+1\right) \leq q\left(\tau_{m}, h_{m}\right)<1,
$$

and we may argue as in the previous case. Specifically, we first observe that

$$
\liminf _{j \rightarrow \infty}\left(\tau_{j}^{m+2}-\tau_{j}^{m+1}\right)>0
$$

and then we see that $q$ and $q^{m}$ satisfy (34) with thresholds $\ell_{m}+1$ and $\ell_{m}$, respectively, which leads to the same contradiction as before. This completes the proof of (b).

We now move to the proof of (c). Suppose that for each $\varepsilon \in(0,1)$ there exists $t<T+\varepsilon$ such that $q^{m}(t) \neq q(t)$. It is clear that the time $\tau_{m+1}$ defined in (31) is smaller than or equal to $T$. Furthermore, if for a given $\varepsilon \in(0,1)$ we had a sequence $\mathcal{J} \subset \mathcal{K}$ such that $\tau_{j}^{m+1} \geq \tau_{m+1}+\varepsilon$ for all $j \in \mathcal{J}$, this would imply that $q^{m}(t)=q(t)$ for all $t \in\left[0, \tau_{m+1}+\varepsilon\right]$, which contradicts (31). Consequently,

$$
\limsup _{k \rightarrow \infty} \tau_{k}^{m+1} \leq \tau_{m+1}
$$

In particular, every subsequence of $\mathcal{K}$ has a further subsequence $\mathcal{J}$ such that $\tau_{j}^{m+1}$ converges to some $\tilde{\tau}_{m+1} \in\left[0, \tau_{m+1}\right]$. To establish the limit appearing in (31) it suffices to show that $\tilde{\tau}_{m+1}=\tau_{m+1}$ in all cases, which can be done as in the proof of (b). Namely, if we assume that $\tilde{\tau}_{m+1}<\tau_{m+1}$, we can show that $q$ and $q^{m}$ satisfy (34) in a right neighborhood of $\tilde{\tau}_{m+1}$ with different thresholds, and this cannot happen because $q^{m}(t)=q(t)$ for all $t \in\left[0, \tau_{m+1}\right]$. Therefore, (31) holds.

To prove (32), we note that $\ell_{k}(t)=\ell_{m}$ for all $t \in\left[\tau_{k}^{m}, \tau_{k}^{m+1}\right.$ ) and all large enough $k \in \mathcal{K}$. In addition, either $q_{k}\left(\tau_{k}^{m+1-}, \ell_{m}\right)=\alpha$ and the threshold decreases at $\tau_{k}^{m+1}$ or $q_{k}\left(\tau_{k}^{m+1-}, h_{m}\right)=1$ and the threshold increases at $\tau_{k}^{m+1}$. Consequently, either $q\left(\tau_{m+1}, \ell_{m}\right)=$ $\alpha$ or $q\left(\tau_{m+1}, h_{m}\right)=1$. In the first case, a threshold decrease occurs at $\tau_{k}^{m+1}$ consistently for all sufficiently large $k \in \mathcal{K}$, so $\ell_{k}\left(\tau_{k}^{m+1}\right)=\ell_{m}-1$ for all large enough $k \in \mathcal{K}$. In the second case, a threshold increase occurs at $\tau_{k}^{m+1}$ consistently for all large enough $k \in \mathcal{K}$, with $\ell_{k}\left(\tau_{k}^{m+1}\right)=\ell_{m}+1$. This establishes (32).

Since $q\left(\tau_{m+1}, \ell_{m}\right)=\alpha$ or $q\left(\tau_{m+1}, h_{m}\right)=1$, we conclude from (30) that $\tau_{m+1}>\tau_{m}$. The other two inequalities in (33) are obtained using (a). The first one holds trivially if $q\left(\tau_{m+1}, h_{m}\right)=1$ and follows from Lemma 24 if $q\left(\tau_{m+1}, \ell_{m}\right)=\alpha$. The second one is a consequence of the fact that $q\left(h_{m}+1\right)$ does not increase along the interval $\left[\tau_{m}, \tau_{m+1}\right)$; this observation is proven as in (ii).

We are now ready to prove Theorem 9 .

Proof. Proof of Theorem 9. Fix $\omega \in \Gamma_{T}^{\ell}$ and recall that this set has probability one. We will prove that every increasing sequence of natural numbers has a subsequence $\mathcal{K}$ such 
that $\left\{s_{k}: k \in \mathcal{K}\right\}$ converges with respect to $\varrho$ to a function $s \in S$ that may be extended to a fluid system.

Given an increasing sequence of natural numbers, we established in Proposition 23 that there exists a subsequence $\mathcal{K}$ such that $\left\{q_{k}: k \in \mathcal{K}\right\}$ and $\left\{q_{k}^{m}: k \in \mathcal{K}\right\}$, for all $m \geq 0$, converge with respect to $\rho_{u}^{\infty}$ over $[0, T+1]$. We denote the limits of these sequences by $q$ and $q^{m}$, respectively.

Assumptions 2 and 3 imply the hypotheses of Lemma 25 hold with $m=0$. We may apply this lemma recursively to construct a sequence of thresholds $\left\{\ell_{m}: 0 \leq m<\theta\right\}$ and a sequence of increasing times $\left\{\tau_{m}: 0 \leq m<\theta\right\}$ such that the following conditions hold; we define $\tau_{\theta}=T$ if the latter sequences have finitely many elements (i.e., if $\theta<\infty$ ).

(i) For all $0 \leq m<\theta$ we have

$$
\dot{q}^{m}(i)=\lambda p_{i}\left(q^{m}, \ell_{m}\right)-i\left[q^{m}(i)-q^{m}(i+1)\right] \quad \text { for all } \quad i \geq 1
$$

and almost everywhere on $\left[\tau_{m}, \tau_{m+1}\right)$. In addition, $q^{m}(t)=q(t)$ for all $t \in\left[\tau_{m}, \tau_{m+1}\right)$.

(ii) For all $0 \leq m<\theta$ we have

$$
\lim _{k \rightarrow \infty} \tau_{k}^{m}=\tau_{m} \in[0, T] \text { and } \lim _{k \rightarrow \infty} \ell_{k}\left(\tau_{k}^{m}\right)=\ell_{m} \in \mathbb{N}
$$

(iii) One of the following holds:

$$
\theta=\infty \quad \text { or } \quad \theta<\infty \quad \text { and } \quad \liminf _{k \rightarrow \infty} \tau_{k}^{\theta}>T
$$

(iv) For all $0 \leq m<\theta-1$ either $q\left(\tau_{m+1}, \ell_{m}\right)=\alpha$ or $q\left(\tau_{m+1}, h_{m}\right)=1$. Moreover,

$$
\ell_{m+1}=\left\{\begin{array}{lll}
\ell_{m}-1 & \text { if } & q\left(\tau_{m+1}, \ell_{m}\right)=\alpha \\
\ell_{m}+1 & \text { if } & q\left(\tau_{m+1}, h_{m}\right)=1
\end{array}\right.
$$

(v) For all $0 \leq m<\theta$ we have

$$
q\left(t, \ell_{m}\right) \geq \alpha \quad \text { for all } t \in\left[\tau_{m}, \tau_{m+1}\right) \quad \text { and } \quad q\left(t, h_{m}\right)<1 \quad \text { a.e. on } \quad\left[\tau_{m}, \tau_{m+1}\right) \text {. }
$$

The first four conditions correspond to assertions in the statement of Lemma 25, whereas the last condition is a consequence of (ii), as we explain below.

Let us fix $0 \leq m<\theta$ to argue that (v) follows from (ii). For the first part of (v) we also fix $t \in\left(\tau_{m}, \tau_{m+1}\right)$ and we pick, for each $k \in \mathcal{K}$, a jump time $\sigma_{k}$ of $\mathcal{N}_{\lambda}(k \cdot)$, so that $\sigma_{k} \rightarrow t$. For all sufficiently large $k$ we have $\sigma_{k} \in\left(\tau_{k}^{m}, \tau_{k}^{m+1}\right)$ and $\ell_{k}\left(\sigma_{k}\right)=\ell_{m}$, which imply $q_{k}\left(\sigma_{k}, \ell_{m}\right)>\alpha$. Taking the limit as $k \rightarrow \infty$ we get $q\left(t, \ell_{m}\right) \geq \alpha$, and by continuity this also holds for $t=\tau_{m}$. For the second part of (v) note that (ii) implies $h_{k}(t)=h_{m}$ 
for all sufficiently large $k \in \mathcal{K}$. Therefore, server pools with at least $h_{m}+1$ tasks do not receive tasks during $\left[\tau_{k}^{m}, \tau_{k}^{m+1}\right)$ for all these $k$. Reasoning as in Lemma 22 we conclude that $p_{i}\left(q(t), \ell_{m}\right)=0$ almost everywhere on $\left[\tau_{m}, \tau_{m+1}\right)$ for all $i \geq h_{m}+1$. Equivalently, $q\left(t, h_{m}\right)<1$ almost everywhere on $\left[\tau_{m}, \tau_{m+1}\right)$.

Above we contemplated the possibility of an infinite $\theta$. However, we now argue that this cannot happen. Specifically, when we apply Lemma 25 recursively to define the update times $\tau_{m}$ and the thresholds $\ell_{m}$, after a finite number of applications, we see that (b) of this lemma holds:

$$
\liminf _{k \rightarrow \infty} \tau_{k}^{m+1}>T
$$

At this point we set $\theta=m+1$ and we conclude the recursion. To understand why this must necessarily happen suppose $\theta$ was infinite. Then we could define

$$
\tau_{\theta}=\lim _{m \rightarrow \infty} \tau_{m}
$$

and we could consider the function $\ell:\left[0, \tau_{\theta}\right) \longrightarrow \mathbb{N}$ such that $\ell(t)=\ell_{m}$ for all $t \in\left[\tau_{m}, \tau_{m+1}\right)$ and all $m \geq 0$. Conditions (i)-(v) would imply that $\left(\left.q\right|_{\left[0, \tau_{\theta}\right)}, \ell\right)$ is a fluid system, so by Proposition 8 we would have $\tau_{\theta}=\infty$. However, this is a contradiction because $\tau_{m} \leq T$ for all $m \geq 0$.

Since $\theta<\infty$ we may define $\ell:[0, T] \longrightarrow \mathbb{N}$ such that

$$
\ell(t)= \begin{cases}\ell_{m} & \text { if } \quad t \in\left[\tau_{m}, \tau_{m+1}\right) \quad \text { and } \quad 0 \leq m<\theta-1 \\ \ell_{\theta-1} & \text { if } \quad t \in\left[\tau_{\theta-1}, T\right] .\end{cases}
$$

Condition (ii) implies that $\left\{\ell_{k}: k \in \mathcal{K}\right\}$ converges to $\ell$ with respect to $\rho_{s}$. Therefore, we conclude that $\left\{s_{k}: k \in \mathcal{K}\right\}$ converges to $s=\left(\left.q\right|_{[0, T]}, \ell\right)$ with respect to $\varrho$.

It only remains to prove that $s$ can be extended to a fluid system. This will be done by extending the sequences $\left\{\ell_{m}: 0 \leq m<\theta\right\}$ and $\left\{\tau_{m}: 0 \leq m<\theta\right\}$ to sequences of $\eta \geq \theta$ elements, and by extending $\left.q\right|_{[0, T]}$ to a function that satisfies (2) with threshold $\ell_{m}$ between $\tau_{m}$ and $\tau_{m+1}$.

We proceed recursively. If $q\left(T, \ell_{\theta-1}\right)=\alpha$ or $q\left(T, h_{\theta-1}\right)=1$, then for the first step we let

$$
\tau_{\theta}=T \quad \text { and } \quad \ell_{\theta}=\left\{\begin{array}{lll}
\ell_{\theta-1}-1 & \text { if } & q\left(T, \ell_{\theta-1}\right)=\alpha \\
\ell_{\theta-1}+1 & \text { if } & q\left(T, h_{\theta-1}\right)=1 .
\end{array}\right.
$$

Otherwise, we fix a solution $\tilde{q}:[0, \infty) \longrightarrow Q$ of $(2)$ with threshold $\ell_{\theta-1}$ and initial condition $q(T)$. We then extend $s$ to a right neighborhood of $T$ as follows.

(a) If $\tilde{q}\left(t, \ell_{\theta-1}\right)>\alpha$ and $\tilde{q}\left(t, h_{\theta-1}\right)<1$ for all $t \geq 0$, then we let $\eta=\theta$ and we extend 
$\left.q\right|_{[0, T]}$ by setting $q(t)=\tilde{q}(t-T)$ for all $t \geq T$. In this case the extension procedure concludes here.

(b) Otherwise, we define $\tau_{\theta}=\inf \left\{t \geq T: \tilde{q}\left(t-T, \ell_{\theta-1}\right)=\alpha\right.$ or $\left.\tilde{q}\left(t-T, h_{\theta-1}\right)=1\right\}$; observe that $\tau_{\theta}>T$ since $\tilde{q}\left(T, \ell_{\theta-1}\right)>\alpha$ and $\tilde{q}\left(T, h_{\theta-1}\right)<1$. In this case we define $\ell_{\theta}$ as above and we extend $\left.q\right|_{[0, T]}$ as in (a), but only to $\left[0, \tau_{\theta}\right]$. Note that $q\left(\tau_{\theta}, \ell_{\theta-1}-1\right)>\alpha$, by Lemma 24 , and that $q\left(\tau_{\theta}, h_{\theta-1}+1\right)<1$, because $q\left(h_{\theta-1}+1\right)$ is non-increasing along $\left[T, \tau_{\theta}\right]$. These two observations imply that $q\left(\tau_{\theta}, \ell_{\theta}\right)>\alpha$ and $q\left(\tau_{\theta}, h_{\theta}\right)<1$. Then we repeat the procedure that we have described to extend $s$ further, to a right neighborhood of $\tau_{\theta}$.

It is possible to check that this procedure results in a fluid system, as defined in Definition 7.

\section{Appendix B Diffusion limits}

In this appendix we prove Theorem 6 and we state and prove an analogous theorem for the case $\lambda \in \mathbb{N}$. We begin by stating the latter theorem. For this purpose, we define:

$$
\hat{Y}_{n}=\sum_{i=1}^{\lambda} \frac{n-Q_{n}(i)}{\sqrt{n}}, \quad \hat{Q}_{n}(i)=\frac{Q_{n}(i)}{\sqrt{n}} \quad \text { for all } \quad i \geq \lambda+1 .
$$

Theorem 26. Suppose $\lambda \in \mathbb{N}, \ell_{n} \equiv \lambda$, there exists $M \geq \lambda+1$ such that $Q_{n}(0, M+1)$ is identically zero for all large enough $n$ and we have the weak convergence

$$
\left(\hat{Y}_{n}(0), \hat{Q}_{n}(0, \lambda+1), \ldots, \hat{Q}_{n}(0, M)\right) \Rightarrow(\hat{Y}(0), \hat{Q}(0, \lambda+1), \ldots, \hat{Q}(0, M)) \quad \text { as } \quad n \rightarrow \infty
$$

Then the process $\left(\hat{Y}_{n}, \hat{Q}_{n}(\lambda+1), \ldots, \hat{Q}_{n}(M)\right)$ converges weakly in the Skorokhod space $D_{\mathbb{R}^{M-\lambda+1}}[0, \infty)$ to the process $(\hat{Y}, \hat{Q}(\lambda+1), \ldots, \hat{Q}(M))$ defined by the following stochastic integral equation:

$$
\begin{aligned}
& \hat{Y}(t)=\hat{Y}(0)+\sqrt{2 \lambda} W(t)+V_{1}(t)-\int_{0}^{t}[\hat{Y}(s)+\lambda \hat{Q}(s, \lambda+1)] d s \\
& \hat{Q}(t, \lambda+1)=\hat{Q}(0, \lambda+1)+V_{1}(t)-\int_{0}^{t}(\lambda+1)[\hat{Q}(s, \lambda+1)-\hat{Q}(s, \lambda+2)] d s \\
& \hat{Q}(t, i)=\hat{Q}(0, i)-\int_{0}^{t} i[\hat{Q}(s, i)-\hat{Q}(s, i+1)] d s, \quad i=\lambda+2, \ldots, M-1 \\
& \hat{Q}(t, M)=\hat{Q}(0, M)-\int_{0}^{t} M \hat{Q}(s, M) d s
\end{aligned}
$$

Here $W$ is a Wiener process and $V_{1}$ is the unique non-decreasing process in $D_{\mathbb{R}}[0, \infty)$ such that

$$
\int_{0}^{t} \mathbb{1}_{\{\hat{Y}(s)>0\}} d V_{1}(s)=0 .
$$


Loosely speaking, this theorem says that, for large enough $n$, and after sufficient time, there will be $O(\sqrt{n})$ server pools with fewer than $\lambda$ or more than $\lambda$ tasks; most of the server pools will have exactly $\lambda$ tasks.

\section{B.1 Proof of the diffusion limits}

The key ingredient of the proofs is a stochastic coupling between a system where tasks are dispatched according to our policy and a system that uses JSQ. For these systems we assume that tasks are blocked if all server pools have $\lfloor\lambda\rfloor+1$ tasks. After establishing this coupling, the results stated above are obtained by invoking the diffusion limits of [23] for JSQ, and by showing that, in non-blocking systems, the probability that tasks are sent to a server pool with $\lfloor\lambda\rfloor+1$ or more tasks over a fixed finite interval of time vanishes as $n \rightarrow \infty$, for both policies.

To describe the coupling, we consider two systems with $n$ server pools each. The first system uses JSQ and its state is denoted $Q_{n}^{1}$, whereas the second system uses our policy with $\ell_{n} \equiv\lfloor\lambda\rfloor$ and its state is denoted $Q_{n}^{2}$. We consider a single Poisson arrival process; each jump of this process corresponds to an arrival in both systems. Also, we use a single Poisson process of potential departures, with rate $(\lfloor\lambda\rfloor+1) n$, to account for service completions. Specifically, we let

$$
a_{n}^{k}=\frac{Q_{n}^{k}(\lfloor\lambda\rfloor+1)}{n} \quad \text { and } \quad b_{n}^{k}=\frac{1}{(\lfloor\lambda\rfloor+1) n} \sum_{i=1}^{\lfloor\lambda\rfloor} Q_{n}^{k}(i) \quad \text { for each } \quad k \in\{1,2\}
$$

and at each potential departure epoch $\tau$ we draw a single random variable $U$, uniform on $(0,1)$. If $U<a_{n}^{k}(\tau)$, then we choose one server pool uniformly at random, among those with exactly $\lfloor\lambda\rfloor+1$ tasks, and we remove one task. If $a_{n}^{k}(\tau) \leq U<a_{n}^{k}(\tau)+b_{n}^{k}(\tau)$ then we also choose one server pool uniformly at random, but among those with fewer than $\lfloor\lambda\rfloor+1$ tasks, and again we remove one task. No changes occur in the remaining case: $a_{n}^{k}(\tau)+b_{n}^{k}(\tau) \leq U$.

Proposition 27. Fix $n$ and suppose $\ell_{n} \equiv\lfloor\lambda\rfloor$. The vector-valued processes

$$
X_{n}^{1}=\left(\sum_{i=1}^{\lfloor\lambda\rfloor} Q_{n}^{1}(i), Q_{n}^{1}(\lfloor\lambda\rfloor+1)\right) \quad \text { and } \quad X_{n}^{2}=\left(\sum_{i=1}^{\lfloor\lambda\rfloor} Q_{n}^{2}(i), Q_{n}^{2}(\lfloor\lambda\rfloor+1)\right)
$$

have the same law provided that the initial conditions, $Q_{n}^{1}(0)$ and $Q_{n}^{2}(0)$, have the same distribution.

Proof. Using the coupling described above we may construct the two systems on a common probability space, with the same initial condition. We will prove that $X_{n}^{1}(t)=X_{n}^{2}(t)$ for all $t \geq 0$ with probability one. To this end we consider the random times $0=\tau_{0}<\tau_{1}<\ldots$ of arrivals or potential departures, and we proceed by induction. By construction $X_{n}^{1}\left(\tau_{0}\right)=$ 
$X_{n}^{2}\left(\tau_{0}\right)$. Suppose this holds at $\tau_{i}$ for some $i \geq 0$, to prove that it holds at $\tau_{i+1}$ as well. We will consider several cases.

First, assume $\tau_{i+1}$ is an arrival epoch. If the first entries $X_{n}^{1}\left(\tau_{i}, 1\right)=X_{n}^{2}\left(\tau_{i}, 1\right)$ are less than $n\lfloor\lambda\rfloor$, then both systems have some server pool with less than $\lfloor\lambda\rfloor$ tasks right before $\tau_{i+1}$. Hence, both systems will assign the new task to a server pool with less than $\lfloor\lambda\rfloor$ tasks. As a result, only the first components of $X_{n}^{1}$ and $X_{n}^{2}$ will change, increasing by one in both cases. Suppose instead that $X_{n}^{1}\left(\tau_{i}, 1\right)=X_{n}^{2}\left(\tau_{i}, 2\right)=n\lfloor\lambda\rfloor$ and $X_{n}^{1}\left(\tau_{i}, 2\right)=X_{n}^{2}\left(\tau_{i}, 2\right)<n$. Then, right before $\tau_{i+1}$, all server pools in both systems are either full or only have one idle server. The new task is sent to one of the idle servers in both systems, and only the second components of $X_{n}^{1}$ and $X_{n}^{2}$ change, increasing by one in both cases. In the remaining case $X_{n}^{1}\left(\tau_{i}\right)=X_{n}^{2}\left(\tau_{i}\right)=(n\lfloor\lambda\rfloor, n)$. Here both systems are completely full right before $\tau_{i+1}$, so the new task has to be discarded. Thus, $X_{n}^{1}$ and $X_{n}^{2}$ do not change.

Finally, suppose that $\tau_{i+1}$ is a potential departure epoch and observe that $X_{n}^{1}\left(\tau_{i}\right)=$ $X_{n}^{2}\left(\tau_{i}\right)$ implies that $a_{n}^{1}\left(\tau_{i}\right)=a_{n}^{2}\left(\tau_{i}\right)$ and $b_{n}^{1}\left(\tau_{i}\right)=b_{n}^{2}\left(\tau_{i}\right)$. As a result, $X_{n}^{1}\left(\tau_{i+1}\right)=X_{n}^{2}\left(\tau_{i+1}\right)$.

We have assumed for simplicity that both systems have no server pools with more than $\lfloor\lambda\rfloor+1$ tasks at all times. However, the stochastic coupling and the previous result can also be established, using similar arguments, if server pools are allowed to have more than $\lfloor\lambda\rfloor+1$ tasks at time zero, but block tasks once they already have $\lfloor\lambda\rfloor+1$ or more tasks.

Consider the processes $\left(\bar{Y}_{n}^{k}, \bar{Z}_{n}^{k}\right)$ and $\left(\hat{Y}_{n}^{k}, \hat{Q}_{n}^{k}(\lambda+1)\right)$ defined by (7) and (35), respectively, for the two truncated systems with finite-size server pools. The law of these processes is independent of $k$ by Proposition 27. The next proposition implies that if these processes have a limit in distribution then their counterparts for the untruncated systems have the same limit, and vice versa.

Proposition 28. Suppose the hypotheses of either Theorem 6 or Theorem 26 hold and fix $T>0$. The probability that a task is dispatched to a server pool with $\lfloor\lambda\rfloor+1$ or more tasks during $[0, T]$ vanishes as $n \rightarrow \infty$ when the threshold policy is used, and the same is true if JSQ is used.

Proof. We prove the result for the threshold policy; the proof for JSQ is similar. To this purpose, let $\tau_{n}$ be the random time at which a task is assigned to a server pool with $\lfloor\lambda\rfloor+1$ or more tasks for the first time. During $\left[0, \tau_{n}\right)$ we have that $\Sigma_{n}=\sum_{i=1}^{\lfloor\lambda\rfloor+1} Q_{n}(i)$ coincides with the total number of tasks in an infinite-server system with $\Sigma_{n}(0)$ tasks at time zero. If $\Sigma_{n}(0)<n(\lambda+\varepsilon)$ for a fixed $\varepsilon<\lfloor\lambda\rfloor+1-\lambda$, then $\Sigma_{n}(t)<n(\lfloor\lambda\rfloor+1)$ during $[0, T]$ with probability tending to one with $n$, and this implies that $\tau_{n}>T$ with probability tending to one. Under the hypotheses of Theorems 6 and 26 the probability of $Q_{n}(0,\lfloor\lambda\rfloor+1)<n(\lambda-\lfloor\lambda\rfloor+\varepsilon)$ converges to one with $n$. Equivalently, the probability of $\Sigma_{n}(0) \geq n(\lambda+\varepsilon)$ vanishes with $n$. 
Theorem 6 now follows from the diffusion limit of [23] for JSQ. Indeed, (a) and (b) only concern the processes $\left(\bar{Y}_{n}, \bar{Z}_{n}\right)$, and (c) follows from Proposition 28 directly. The stochastic dynamics in the statement of Theorem 26 involve not only $\hat{Y}$ and $\hat{Q}(\lambda+1)$ but also $\hat{Q}(i)$ for $i=\lambda+2, \ldots, M$. However, as in [10, Section 5.2], the dynamics of $(\hat{Q}(\lambda+2), \ldots, \hat{Q}(M))$ are deterministic since tasks are not sent to server pools with $\lambda+1$ or more tasks with probability tending to one. The proof of Theorem 26 is completed by this observation and the diffusion limit of [23] for JSQ.

\section{References}

[1] F. Baccelli, B. Kauffmann, and D. Veitch, "Inverse problems in queueing theory and internet probing," Queueing Systems, vol. 63, no. 1-4, p. 59, 2009.

[2] R. Badonnel and M. Burgess, "Dynamic pull-based load balancing for autonomic servers," in NOMS 2008-2008 IEEE Network Operations and Management Symposium. IEEE, 2008, pp. 751-754.

[3] N. Benameur, S. B. Fredj, S. Oueslati-Boulahia, and J. W. Roberts, "Quality of service and flow level admission control in the internet," Computer Networks, vol. 40, no. 1, pp. 57-71, 2002.

[4] P. Billingsley, Convergence of probability measures. John Wiley \& Sons, 2013.

[5] T. Bonald, M. Jonckheere, and A. Proutière, "Insensitive load balancing," ACM Sigmetrics Performance Evaluation Review, vol. 32, no. 1, pp. 367-377, 2004.

[6] M. Bramson, "State space collapse with application to heavy traffic limits for multiclass queueing networks," Queueing Systems, vol. 30, no. 1-2, pp. 89-140, 1998.

[7] C. Comte, "Dynamic load balancing with tokens," Computer communications, vol. 144, pp. 76-88, 2019.

[8] M. Defraeye and I. Van Nieuwenhuyse, "Staffing and scheduling under nonstationary demand for service: A literature review," Omega, vol. 58, pp. 4-25, 2016.

[9] A. Ephremides, P. Varaiya, and J. Walrand, "A simple dynamic routing problem," IEEE transactions on Automatic Control, vol. 25, no. 4, pp. 690-693, 1980.

[10] P. Eschenfeldt and D. Gamarnik, "Join the shortest queue with many servers. the heavy-traffic asymptotics," Mathematics of Operations Research, vol. 43, no. 3, pp. 867-886, 2018.

[11] D. Gamarnik, J. N. Tsitsiklis, and M. Zubeldia, "Delay, memory, and messaging tradeoffs in distributed service systems," Stochastic Systems, vol. 8, no. 1, pp. 45-74, 2018. 
[12] D. Goldsztajn, A. Ferragut, and F. Paganini, "Feedback control of server instances for right sizing in the cloud," in 2018 56th Annual Allerton Conference on Communication, Control, and Computing (Allerton). IEEE, 2018, pp. 749-756.

[13] D. Goldsztajn, A. Ferragut, F. Paganini, and M. Jonckheere, "Controlling the number of active instances in a cloud environment," ACM SIGMETRICS Performance Evaluation Review, vol. 45, no. 3, pp. 15-20, 2018.

[14] V. Gupta and M. Harchol-Balter, "Self-adaptive admission control policies for resource-sharing systems," in Proceedings of the eleventh international joint conference on Measurement and modeling of computer systems, 2009, pp. 311-322.

[15] I. A. Horváth, Z. Scully, and B. Van Houdt, "Mean field analysis of join-belowthreshold load balancing for resource sharing servers," Proceedings of the ACM on Measurement and Analysis of Computing Systems, vol. 3, no. 3, pp. 1-21, 2019.

[16] M. Jonckheere and B. J. Prabhu, "Asymptotics of insensitive load balancing and blocking phases," in Proceedings of the 2016 ACM SIGMETRICS International Conference on Measurement and Modeling of Computer Science, 2016, pp. 311-322.

[17] A. Karthik, A. Mukhopadhyay, and R. R. Mazumdar, "Choosing among heterogeneous server clouds," Queueing Systems, vol. 85, no. 1-2, pp. 1-29, 2017.

[18] P. Key, L. Massoulié, A. Bain, and F. Kelly, "Fair internet traffic integration: network flow models and analysis," in Annales des Telecommunications, vol. 59, no. 11. Springer, 2004, pp. 1338-1352.

[19] Y. Lu, Q. Xie, G. Kliot, A. Geller, J. R. Larus, and A. Greenberg, "Join-idle-queue: A novel load balancing algorithm for dynamically scalable web services," Performance Evaluation, vol. 68, no. 11, pp. 1056-1071, 2011.

[20] R. Menich and R. F. Serfozo, "Optimality of routing and servicing in dependent parallel processing systems," Queueing Systems, vol. 9, no. 4, pp. 403-418, 1991.

[21] M. Mitzenmacher, "The power of two choices in randomized load balancing," IEEE Transactions on Parallel and Distributed Systems, vol. 12, no. 10, pp. 1094-1104, 2001.

[22] D. Mukherjee, S. C. Borst, J. S. Van Leeuwaarden, and P. A. Whiting, "Universality of load balancing schemes on the diffusion scale," Journal of Applied Probability, vol. 53, no. 4, pp. 1111-1124, 2016.

[23] _ - "Asymptotic optimality of power-of-d load balancing in large-scale systems," Mathematics of Operations Research, vol. 45, no. 4, pp. 1535-1571, 2020. 
[24] D. Mukherjee, S. Dhara, S. C. Borst, and J. S. Van Leeuwaarden, "Optimal service elasticity in large-scale distributed systems," Proceedings of the ACM on Measurement and Analysis of Computing Systems, vol. 1, no. 1, pp. 1-28, 2017.

[25] A. Mukhopadhyay, A. Karthik, R. R. Mazumdar, and F. Guillemin, "Mean field and propagation of chaos in multi-class heterogeneous loss models," Performance Evaluation, vol. 91, pp. 117-131, 2015.

[26] A. Mukhopadhyay, R. R. Mazumdar, and F. Guillemin, "The power of randomized routing in heterogeneous loss systems," in 2015 27th International Teletraffic Congress. IEEE, 2015, pp. 125-133.

[27] P. D. Sparaggis, D. Towsley, and C. Cassandras, "Extremal properties of the shortest/longest non-full queue policies in finite-capacity systems with state-dependent service rates," Journal of Applied Probability, pp. 223-236, 1993.

[28] A. L. Stolyar, "Pull-based load distribution in large-scale heterogeneous service systems," Queueing Systems, vol. 80, no. 4, pp. 341-361, 2015.

[29] J. N. Tsitsiklis and K. Xu, "On the power of (even a little) resource pooling," Stochastic Systems, vol. 2, no. 1, pp. 1-66, 2013.

[30] S. R. Turner, "The effect of increasing routing choice on resource pooling," Probability in the Engineering and Informational Sciences, vol. 12, no. 1, pp. 109-124, 1998.

[31] M. Van der Boor, S. C. Borst, J. S. Van Leeuwaarden, and D. Mukherjee, "Scalable load balancing in networked systems: A survey of recent advances," arXiv preprint arXiv:1806.05444, 2018.

[32] N. D. Vvedenskaya, R. L. Dobrushin, and F. I. Karpelevich, "Queueing system with selection of the shortest of two queues: An asymptotic approach," Problemy Peredachi Informatsii, vol. 32, no. 1, pp. 20-34, 1996.

[33] A. Wierman, L. L. Andrew, and A. Tang, "Power-aware speed scaling in processor sharing systems: Optimality and robustness," Performance Evaluation, vol. 69, no. 12, pp. 601-622, 2012.

[34] W. Winston, "Optimality of the shortest line discipline," Journal of Applied Probability, pp. 181-189, 1977.

[35] Q. Xie, X. Dong, Y. Lu, and R. Srikant, "Power of d choices for large-scale bin packing: A loss model," ACM SIGMETRICS Performance Evaluation Review, vol. 43, no. 1, pp. 321-334, 2015. 
[36] F. Yao, A. Demers, and S. Shenker, "A scheduling model for reduced cpu energy," in Proceedings of IEEE 36th annual foundations of computer science. IEEE, 1995, pp. $374-382$.

[37] X. Zhou, J. Tan, and N. Shroff, "Heavy-traffic delay optimality in pull-based load balancing systems: Necessary and sufficient conditions," Proceedings of the ACM on Measurement and Analysis of Computing Systems, vol. 2, no. 3, pp. 1-33, 2018.

[38] X. Zhou, F. Wu, J. Tan, Y. Sun, and N. Shroff, "Designing low-complexity heavytraffic delay-optimal load balancing schemes: Theory to algorithms," Proceedings of the ACM on Measurement and Analysis of Computing Systems, vol. 1, no. 2, pp. 1-30, 2017 .

[39] M. Zubeldia, "Delay, stability, and resource tradeoffs in large distributed service systems," Ph.D. dissertation, Massachusets Institute of Technology, 2019. 